\title{
On the vanishing of coefficients of the powers of a theta function
}

\author{
Jacques Sauloy, Changgui Zhang ${ }^{\dagger}$
}

3 août 2020

\begin{abstract}
A result on the Galois theory of $q$-difference equations [3] leads to the following question: if $q \in \mathbf{C}^{*},|q|<1$ and if one sets $\theta_{q}(z):=\sum_{m \in \mathbf{Z}} q^{m(m-1) / 2} z^{m}$, can some coefficients of the Laurent series expansion of $\theta_{q}^{k}(z), k \in \mathbf{N}^{*}$, vanish ? We give a partial answer.
\end{abstract}

\section{Introduction}

\subsection{Origin of the problem}

Let $q \in \mathbf{C}$ such that $|q|>1$. In [3], one uses the function 1$] \theta_{q}(z):=\sum_{m \in \mathbf{Z}} q^{-m(m+1) / 2} z^{m}$, $z \in \mathbf{C}^{*}$. This is a holomorphic function over $\mathbf{C}^{*}$. For every $k \in \mathbf{N}^{*}$, one introduces the coefficients $t_{n}^{(k)}, n \in \mathbf{Z}$, through the expansion into a Laurent series $\theta_{q}^{k}(z)=\sum t_{n}^{(k)} z^{n}$. These series converge normally over every compact subset of the domain $\mathbf{C} \backslash \overline{\mathbf{D}}(0,1)$, whence the explicit formula:

$$
t_{n}^{(k)}=\sum_{\substack{m_{1}, \ldots, m_{k} \in \mathbf{Z} \\ m_{1}+\cdots+m_{k}=n}} q^{-\left(m_{1}\left(m_{1}+1\right)+\cdots+m_{k}\left(m_{k}+1\right)\right) / 2},
$$

which implies that each $t_{n}^{(k)}$ is, as a function of $q$, holomorphic over the domain $\mathbf{C} \backslash \overline{\mathbf{D}}(0,1)$. Since $q>0 \Rightarrow t_{n}^{(k)}(q)>0$, the function $t_{n}^{(k)}$ is not identically 0 , so the set of its zeroes is

${ }^{*}$ E-mail address:jacques.sauloy@ gmail.com, URL: http://www.cantoperdic.fr/

†Laboratoire P. Painlevé (UMR-CNRS 8524), UFR Math., Université de Lille 1, Cité scientifique, 59655 Villeneuve d'Ascq cedex, France, E-mail address:changgui.zhang@univ-lille.fr, URL:https://math.univlille1.fr/ zhang/

${ }^{1}$ Note however that the conventions in the present work are different, one assumes that $0<|q|<1$ and it is the formula (1) herebelow which defines $\theta_{q}$. Also, notation for the coefficients will differ, see formula (2). 
discrete, whence denumerable. As a consequence, the set of all zeroes of all functions $t_{n}^{(k)}$ is denumerable.

For all $q$ such that no $t_{n}^{(k)}(q)$ vanishes, the first author has described in [3] the "wild fundamental group" of analytic complex linear $q$-difference equations which are irregular at 0 (and with arbitrary slopes). This led to call "good values" of $q$ such values. As we just saw, this property is generically satisfied. The goal of the present article is to study various cases of vanishing of $t_{n}^{(k)}(q)$.

However, to stick as much as possible to the classical litterature on $q$-special functions (above all Ramanujan's opus!), we shall assume in this work that $|q|<1, q \neq 0$, and one shall begin with the following definition:

$$
\theta_{q}(x):=\sum_{m \in \mathbf{Z}} q^{m(m-1) / 2} x^{m}, \quad x \in \mathbf{C}^{*},
$$

which again defines a holomorphic function over $\mathbf{C}^{*}$. For every $k \in \mathbf{N}^{*}$, we shall denote $\gamma_{k, n}, n \in \mathbf{Z}$ the coefficients of the Laurent series expansion of $\theta_{q}^{k}$ :

$$
\theta_{q}^{k}(q)=\sum_{n \in \mathbf{Z}} \gamma_{k, n} q^{n} \Longrightarrow \gamma_{k, n}(q)=\gamma_{k, n}=\sum_{\substack{m_{1}, \ldots, m_{k} \in \mathbf{Z} \\ m_{1}+\cdots+m_{k}=n}} q^{\left(m_{1}\left(m_{1}-1\right)+\cdots+m_{k}\left(m_{k}-1\right)\right) / 2}
$$

The relation to the formulas in [3] is clear: if $|q|>1$ and if $p:=q^{-1}$, then $\theta_{q}(z)=$ $\theta_{p}(x)$ with $x:=p z$, and moreover $t_{n}^{(k)}(q)=\gamma_{k, n}\left(q^{-1}\right)$; and the "good values" of $q,|q|>1$, are those such that none of the $\gamma_{k, n}\left(q^{-1}\right)$ vanishes.

In this work, we shall first study $\theta_{q},|q|<1, q \neq 0$, and the coefficients $\gamma_{k, n}$. However, in 2.2 and then again in 3.2 we shall rather use (for simplicity of the computations) another of the classical theta functions:

$$
\vartheta(x):=\vartheta_{q}(x):=\sum_{n \in \mathbf{Z}} q^{n^{2} / 2} x^{n}
$$

We correspondingly introduce the coefficients of powers of $\vartheta$ :

$$
\vartheta_{q}^{k}(z)=\sum_{n \in \mathbf{Z}} c_{k, n} z^{n} \Longrightarrow c_{k, n}(q)=c_{k, n}=\sum_{\substack{\left(n_{1}, \ldots, n_{k}\right) \in \mathbf{Z}^{k} \\ n_{1}+\cdots+n_{k}=n}} q^{\left(n_{1}^{2}+\cdots+n_{k}^{2}\right) / 2} .
$$

From the obvious relations:

$$
\vartheta(x)=\theta_{q}\left(q^{1 / 2} x\right), \text { whence } c_{k, n}(q)=q^{n / 2} \gamma_{k, n},
$$

we see how both studies are easily related. Our main results consist in a series of sufficient conditions on $k, n$ for the existence of a zero of $\gamma_{k, n}$ or, equivalently, of $c_{k, n}$, in the punctured open disk $\stackrel{\circ}{\mathbf{D}}(0,1) \backslash\{0\}$. However we confess that we could not solve the main mystery: which are the bad values of $q$ ?

\footnotetext{
${ }^{2}$ This definition requires an unambiguous determination of $q^{1 / 2}$, the necessary conventions are explained in 2.2.1.
} 


\subsection{First computations}

Obviously $\gamma_{1, n}(q)=q^{n(n-1) / 2}$ never vanishes. The same is true for $\gamma_{2, n}(q)$ thanks to the following calculation:

$$
\begin{aligned}
\gamma_{2, n}(q) & =\sum_{l+m=n} q^{(l(l-1)+m(m-1)) / 2}=\sum_{l+m=n} q^{n(n-1) / 2} q^{-l m}=q^{n(n-1) / 2} \sum_{m} q^{m(m-n)} \\
& =q^{n(n-1) / 2} \sum_{m}\left(q^{2}\right)^{m(m-1) / 2}\left(q^{-n+1}\right)^{m}=q^{n(n-1) / 2} \theta_{q^{2}}\left(q^{-n+1}\right),
\end{aligned}
$$

which can only vanish for $0<|q|<1$ by virtue of the triple product formula along with the impossibility of relation $-q^{-n+1} \in q^{\mathbf{Z}}$.

Computing $\gamma_{2, n}$ easily yields a closed formula for $\theta_{q}^{2}$ :

$$
\theta_{q}^{2}(x)=\theta_{q^{2}}(q) \theta_{q^{2}}\left(x^{2}\right)+x \theta_{q^{2}}(1) \theta_{q^{2}}\left(q x^{2}\right) .
$$

Here is a direct argument (more general arguments will come later, see sections 2.1 and 2.2):

$$
\begin{aligned}
\theta_{q}^{2}(x) & =\sum_{n \in \mathbf{Z}} \gamma_{2, n}(q) x^{n}=\sum_{n \in \mathbf{Z}} q^{n(n-1) / 2} \theta_{q^{2}}\left(q^{-n+1}\right) x^{n} \\
& =\sum_{m \in \mathbf{Z}} q^{m(2 m-1)} \theta_{q^{2}}\left(q^{-2 m+1}\right) x^{2 m}+\sum_{m \in \mathbf{Z}} q^{m(2 m+1)} \theta_{q^{2}}\left(q^{-2 m}\right) x^{2 m+1} \\
& =\sum_{m \in \mathbf{Z}} q^{m(2 m-1)} q^{-m(m+1)} q^{m} \theta_{q^{2}}(q) x^{2 m}+\sum_{m \in \mathbf{Z}} q^{m(2 m+1)} q^{-m(m+1)} \theta_{q^{2}}(1) x^{2 m+1} \\
& =\theta_{q^{2}}(q) \sum_{m \in \mathbf{Z}} q^{m(m-1)} x^{2 m}+\theta_{q^{2}}(1) \sum_{m \in \mathbf{Z}} q^{m^{2}} x^{2 m+1}=\theta_{q^{2}}(q) \theta_{q^{2}}\left(x^{2}\right)+x \theta_{q^{2}}(1) \theta_{q^{2}}\left(q x^{2}\right) .
\end{aligned}
$$

We used en passant the relation $\theta_{q}\left(q^{-m} x\right)=q^{-m(m+1) / 2} x^{m} \theta_{q}(x)$ (with $q \leftarrow q^{2}$ ).

It is difficult not to hope that such an identity can be extended to higher powers. According to Professor Bruce Berndt, powers of theta functions are tackled in volume III of the "Ramanujan Notebooks" [1]; and indeed, he there gives the proof (Entry 29) of such a relation from which the above equality flows easily; and Entry 30 seems to give the clue to the calculation of $\theta_{q}^{3}(z)$ and maybe of $\theta_{q}^{4}(z)$, but it is not quite clear. At any rate, using Entries 18 to 30 does allow a first approach to our problem, see section 2.1.

Another kind of calculus, akin to the theory of divergent $q$-series [5] and of their transformations ( $q$-Borel, $q$-Laplace ...), allows one to iteratively obtain such identities. We expound it in section 2.2. With a bit of real analysis one can then deduce for instance that $\gamma_{3,0}(q)$ vanishes for at least one negative value of $q$. Using some numerical/formal calculus with Maple, one can make the value more precise. Note that this section rather uses function $\vartheta$ in order to simplify the form of the identities; translating back the results is immediate. 
It is possible to recognize in the series such as that giving $\gamma_{3,0}(q)$ the generating series of the number of representations by some quadratic form:

$$
\gamma_{3,0}(q)=\sum_{\substack{a, b, c \in \mathbf{Z} \\ a+b+c=0}} q^{\left(a^{2}-a+b^{2}-b+c^{2}-c\right) / 2}=\sum_{\substack{a, b, c \in \mathbf{Z} \\ a+b+c=0}} q^{-(a b+b c+c a)}=\sum_{a, b \in \mathbf{Z}} q^{a^{2}+a b+b^{2}}=f(q),
$$

where we have set $f(x):=\sum_{a, b \in \mathbf{Z}} x^{a^{2}+a b+b^{2}}=\sum_{n \geq 0} r(n) x^{n}$ for $|x|<1$; here $r(n)$ denotes the number of pairs $(a, b) \in \mathbf{Z} \times \mathbf{Z}$ such that $a^{2}+a b+b^{2}=n$ (the computation is wholly justified by the fact that this is a positive definite quadratic form). The integer:

$$
R(n):=r(0)+\cdots+r(n)=\operatorname{card}\left\{(a, b) \in \mathbf{Z} \times \mathbf{Z} \mid a^{2}+a b+b^{2} \leq n\right\}
$$

is equivalent, when $n \rightarrow+\infty$, to $C n$, where $C$ is the area of the ellipse $x^{2}+x y+y^{2} \leq 1$, i.e. $2 \pi / \sqrt{3}$. By standard abelian theorems we draw that, when $x \rightarrow 1^{-}$:

$$
\frac{f(x)}{1-x}=\sum_{n \geq 0} R(n) x^{n} \sim \sum C n x^{n}=C \frac{x}{(1-x)^{2}} \Longrightarrow f(x) \sim \frac{C}{1-x} .
$$

On the other hand, the exponent $a^{2}+a b+b^{2}$ is even if, and only if $a$ and $b$ are. The even part of series $f(x)$ is therefore the sub-sum on the such pairs, that is $f\left(x^{4}\right)$, so that $f(-x)=2 f\left(x^{4}\right)-f(x)$. Hence, still for $x \rightarrow 1^{-}$:

$$
f\left(x^{4}\right) \sim \frac{C}{1-x^{4}} \sim \frac{C}{4(1-x)} \Longrightarrow f(-x) \sim \frac{-C}{2(1-x)} \Longrightarrow \lim _{\substack{x \rightarrow-1 \\ x>-1}} f(x)=-\infty .
$$

Since $f(0)=1$, the function $f(x)$, thus also the function $\gamma_{3,0}(q)$, vanishes at least once on ]$-1,0[$. One of our goals is to generalize this fact and the ideas which led to it.

\subsection{A bit of theory}

\subsubsection{Linear relations among theta powers and theta functions}

We shall write various sets of integers as:

$$
\mathbf{N}=\{0,1, \ldots\}, \quad \mathbf{N}^{*}=\mathbf{N} \backslash\{0\}=\{1,2, \ldots\}, \quad \mathbf{Z}=\mathbf{N} \cup(-\mathbf{N})=\{0,1,-1,2,-2, \ldots\} .
$$

Let $k \in \mathbf{N}^{*}$. The $\mathbf{C}$-linear space of holomorphic functions over $\mathbf{C}^{*}$ such that $f(q x)=$ $x^{-k} f(x)$ has dimension $k$, as can be seen by expanding $f$ into a Laurent series $\sum f_{m} x^{m}$ : the latter is uniquely and linearly determined by coefficients $f_{0}, \ldots, f_{k-1}$. The functions $x^{i} \theta_{q^{k}}\left(q^{i} x^{k}\right), i=0, \ldots, k-1$, belong to that space and are linearly independent (their supports are pairwise disjoint). The function $\theta_{q}^{k}$ also belongs to that space, whence a relation:

$$
\theta_{q}^{k}(x)=\sum_{i=0}^{k-1} a_{k, i} x^{i} \theta_{q^{k}}\left(q^{i} x^{k}\right)
$$


For instance identity (5) at the end of the calculation at the beginning of 1.2 says that $a_{2,0}=\theta_{q^{2}}(q)$ and $a_{2,1}=\theta_{q^{2}}(1)$.

Expanding the left hand side along with each term of the right hand side yields the relations:

$$
n=j k+i, 0 \leq i \leq k-1 \Longrightarrow \gamma_{k, n}=\left(q^{k}\right)^{j(j-1) / 2} q^{i j} a_{k, i}
$$

In particular $a_{k, i}=\gamma_{k, i}(i=0, \ldots, k-1)$ so our identity above boils down to:

$$
\theta_{q}^{k}(x)=\sum_{i=0}^{k-1} \gamma_{k, i} x^{i} \theta_{q^{k}}\left(q^{i} x^{k}\right)
$$

Note that the functional equation $\theta_{q}^{k}(q x)=x^{-k} \theta_{q}^{k}(x)$ immediately yields $\gamma_{k, n+k}=q^{n} \gamma_{k, n}$ which implies, by iteration, formula (7).

Thus, on the one hand vanishing of some $\gamma_{k, n}(q)$ only depends of the class of $n$ modulo $k$; on the other hand, the $k$ complex numbers $a_{k, i}, i=0, \ldots, k-1$ contain all relevant information. We are therefore led to look for explicit identities (i.e. with known coefficients) of the above type.

\subsubsection{Some easy facts about the coefficients}

We collect here some facts about the coefficients $\gamma_{k, n}$ (and therefore also about the coefficients $c_{k, n}$ ) which follow directly from the explicit formulas (2) and (4).

First note that $n(n-1) \geq 0$ for all $n \in \mathbf{Z}$, and that it vanishes only for $n=0,1$. So $\gamma_{k, n}(q)$ involves only non negative powers of $q$ and the power $q^{0}$ comes from terms with multiindex $\left(m_{1}, \ldots, m_{k}\right) \in\{0,1\}^{k}$ such that $m_{1}+\cdots+m_{k}=n$. There are $\left(\begin{array}{l}k \\ n\end{array}\right)$ such terms, whence:

$$
\forall k \in \mathbf{N}^{*}, \forall n \in \mathbf{Z}, \gamma_{k, n}(q)=\left(\begin{array}{l}
k \\
n
\end{array}\right)+O(q)
$$

Second, note that in the summation (2), changing $\left(m_{1}, \ldots, m_{k}\right)$ to $\left(1-m_{1}, \ldots, 1-m_{k}\right)$ does not change the general term $q^{(\cdots)}$, permutes $\mathbf{Z}^{k}$ and transforms the sum $n$ into $k-n$. Therefore:

$$
\forall k \in \mathbf{N}^{*}, \forall n \in \mathbf{Z}, \gamma_{k, n}=\gamma_{k, k-n} .
$$

Last, we give without proof an identity somehow related to the above symmetry; it can be checked by elementary manipulation of indexes:

$$
\forall k \in \mathbf{N}^{*}, \forall n \in\{1, \ldots, k-1\}, \gamma_{k, n}=\sum_{m \in \mathbf{Z}} \gamma_{n, m} \gamma_{k-n, m} .
$$




\subsection{Contents of the paper}

In order to have information on the coefficients, we first look for identities in section 2 . Using Ramanujan's formulas in 2.1 gives some information on powers of $\theta_{q}$, unhappily not enough.

So we develop in 2.2 a calculus inspired from the theory of transformations of divergent $q$-series [5]. However, this is more simply done using function $\vartheta$ instead of $\theta_{q}$ (but the results are quite easy to translate). This calculus allows us to find some preliminary information on the vanishing of coefficients with some real analysis.

In 3 we generalize the real analysis argument through an asymptotic study of the coefficients of $\theta_{q}{ }^{k}$, resp. $\vartheta^{k}$, near $q=-1$.

First, in 3.1 we relate said coefficients to generating series of the numbers of representations by quadratic forms. Usual considerations of geometry of numbers yield rather good estimations (theorem 10 and a first list of sufficient conditions for vanishing coefficients (corollary 2). Last, well known modular properties of the generating series allow for some complementary information (proposition 7).

Last, in 3.2 we return to the formalism for $\vartheta$ introduced in 2.2 and use the classical modular transformation formula to obtain again asymptotic estimates (theorems 3 and 2) from which we deduce more sufficient conditions: actually, exactly what is needed to recover corollary 2 and its consequences.

The difference between the two approaches towards the same sufficient conditions is reflected in the nature of the asymptotic estimates: one is in terms of powers of $t=$ $\log \left(e^{-\pi \mathrm{i}} q\right)$, the other is a transseries in the exponential scale.

Note that in the end one mysterious question remains unsolved: what can be said of the "bad values" of $q$ ? Contrary to our first guesses, they are not all real negative ...

\section{Acknowledgements}

The first author is indebted to Professor Berndt for suggesting to look at Ramanujan's Notebooks and positive comments on first attempts.

The second author was supported by Labex CEMPI (Centre Européen pour les Mathématiques, la Physique et leurs Interaction).

\section{Some more notations}

We already introduced $\mathbf{N}, \mathbf{N}^{*}, \mathbf{Z}$ at the beginning of 1.3.1.

For every $k \in \mathbf{N}^{*}$ and $a \in \mathbf{Z}$, we write $a(\bmod k)$ either for the remainder of the euclidean division of $a$ by $k$ (thus $a(\bmod k) \in\{0, \ldots, k-1\})$ or for the congruence class of $a$ modulo $k$ (thus $a(\bmod k) \in \mathbf{Z} / k \mathbf{Z})$. The context should hopefully lift any ambiguity. 
In the same spirit, if $f: \mathbf{Z} \rightarrow \mathbf{C}$ is any $k$-periodic function, one writes $\sum_{a(\bmod k)} f(a)$ a sum taken over an arbitrary set of representatives $a$ modulo $k$.

Last, we shall write in underlined characters $k$-uples $\underline{n}=\left(n_{1}, \ldots, n_{k}\right)$, whether in $\mathbf{Z}^{k}, \mathbf{R}^{k}$

\section{Identities and coefficients}

\subsection{Some identities drawn from "Ramanujan Notebooks"}

Our sole reference in this section is chapter 1 of volume III of the Ramanujan Notebooks [1]. For non zero complex numbers $a, b$ such that $|a b|<1$, Ramanujan introduces the function:

$$
f(a, b):=1+\sum_{k=1}^{+\infty}(a b)^{k(k-1) / 2}\left(a^{k}+b^{k}\right)=\sum_{k=-\infty}^{+\infty} a^{k(k+1) / 2} b^{k(k-1) / 2},
$$

in which can be recognized, in a very symmetric guise, our function $\theta_{q}$. Indeed, setting $q:=a b$, one immediately sees that:

$$
f(a, b)=\theta_{q}(a)
$$

We shall translate in terms of $\theta_{q}$ some identities of Ramanujan (among those for which this is not already done in [1]) then draw consequences.

\subsubsection{Translation of some identities from [1]}

We give no proof of these translations, verifications are mechanical (even if the proof of the identities themselves are not !). Actually, Entry 22 is directly copied from [1].

We omitted the important Entries 20 and 27, which express modularity, because we do not take advantage of that property in the present section (it will appear in section 3).

Entry (18). 1. $\theta_{q}(x)=\theta_{q}(q / x)$

2. $\theta_{q}(q)=2 \theta_{q^{4}}(q)=2 \theta_{q^{4}}\left(q^{3}\right)$

3. $\theta_{q}(-1)=\theta_{q}(-q)=0$

4. $\theta_{q}(x)=q^{n(n-1) / 2} x^{n} \theta_{q}\left(q^{n} x\right)$

The above equalities are elementary; the following is Jacobi's celebrated Triple Product Formula. Recall the Pochhammer symbols $(x ; q)_{\infty}:=\prod_{n \geq 0}\left(1-x q^{n}\right)$.

Entry (19). $\theta_{q}(x)=(q ; q)_{\infty}(-x ; q)_{\infty}(-q / x ; q)_{\infty}$ 
$\operatorname{Entry}(22) . \quad$ 1. $\phi(q):=\theta_{q^{2}}(q)=\sum_{k \in \mathbf{Z}} q^{k^{2}}=\frac{\left(-q ; q^{2}\right)_{\infty}\left(q^{2} ; q^{2}\right)_{\infty}}{\left(q ; q^{2}\right)_{\infty}\left(-q^{2} ; q^{2}\right)_{\infty}}$

2. $\psi(q):=\theta_{q^{4}}(q)=\sum_{k=0}^{\infty} q^{k(k+1) / 2}=\frac{\left(q^{2} ; q^{2}\right)_{\infty}}{\left(q ; q^{2}\right)_{\infty}}$

3. $f(-q):=\theta_{q^{3}}(-q)=\sum_{k \in \mathbf{Z}}(-1)^{k} q^{k(3 k-1) / 2}=(q ; q)_{\infty}$

4. $\chi(q):=\left(-q ; q^{2}\right)_{\infty}$ (note that this item is purely a definition)

The following one is related to the distribution formula of Pochhammer symbols: $\prod_{k=0}^{n-1}\left(q^{k} x ; q^{n}\right)_{\infty}=(x ; q)_{\infty}$.

Entry (28). $\prod_{k=0}^{n-1} \theta_{q^{n}}\left(q^{k} x\right)=\frac{\left(q^{n} ; q^{n}\right)_{\infty}^{n}}{(q ; q)_{\infty}} \theta_{q}(x)$

Entry 29 has been the most fuitful for our goals. The apparent dissymmetry in $x, y$ disappears if one takes in account Entry 18.

Entry (29). 1. $\theta_{q}(x) \theta_{q}(y)+\theta_{q}(-x) \theta_{q}(-y)=2 \theta_{q^{2}}(x y) \theta_{q^{2}}(q y / x)$

2. $\theta_{q}(x) \theta_{q}(y)-\theta_{q}(-x) \theta_{q}(-y)=2 x \theta_{q^{2}}(q x y) \theta_{q^{2}}(y / x)$

Entry (30). 1. $\theta_{q^{2}}(x) \theta_{q^{2}}(q x)=\theta_{q}(x) \psi(q)$

2. $\theta_{q}(x)+\theta_{q}(-x)=2 \theta_{q^{4}}\left(q x^{2}\right)$

3. $\theta_{q}(x)-\theta_{q}(-x)=2 x \theta_{q^{4}}\left(q^{3} x^{2}\right)$

4. $\theta_{q}(x) \theta_{q}(-x)=\theta_{q^{2}}\left(-x^{2}\right) \phi(-q)$

5. $\theta_{q}^{2}(x)+\theta_{q}^{2}(-x)=2 \theta_{q^{2}}\left(x^{2}\right) \phi(q)$

6. $\theta_{q}^{2}(x)-\theta_{q}^{2}(-x)=4 x \theta_{q^{2}}\left(q x^{2}\right) \psi\left(q^{2}\right)$

The following corollary of Entry 30 (actually, seing its proof, rather of Entry 29) looks promising:

$\theta_{q}(x) \theta_{q}(y) \theta_{q}(n x) \theta_{q}(n y)+\theta_{q}(-x) \theta_{q}(-y) \theta_{q}(-n x) \theta_{q}(-n y)=2 x \theta_{q}(y / x) \theta_{q}(n x y) \theta_{q}(n) \psi(q)$.

However, to exploit it, one would have wanted a formula involving the difference

$$
\theta_{q}(x) \theta_{q}(y) \theta_{q}(n x) \theta_{q}(n y)-\theta_{q}(-x) \theta_{q}(-y) \theta_{q}(-n x) \theta_{q}(-n y)
$$

and we found no simple such formula.

At any rate, it seems difficult to find in [1] equalities involving products of functions $\theta_{q}$ with different bases $q$, which explains in part the limited range of the following consequences with respect to our goals. 


\subsubsection{Consequences for the powers of $\theta_{q}$}

One first proves anew identity (5):

\section{Proposition 1.}

$$
\theta_{q}^{2}(x)=\theta_{q^{2}}(q) \theta_{q^{2}}\left(x^{2}\right)+x \theta_{q^{2}}(1) \theta_{q^{2}}\left(q x^{2}\right) .
$$

Proof. The most direct path is to take the arithmetical mean of both items of Entry 29, with $y=x$. One can also take the arithmetical mean of the last two items of Entry 30, then replace $\phi(q)$ and $\psi\left(q^{2}\right)$ according to the two first items of Entry 22, last apply the first two items of Entry 18.

As already noted, $a_{2,0}=\theta_{q^{2}}(q)$ et $a_{2,1}=\theta_{q^{2}}(1)$ (notations of 1.3).

\section{Corollary 1.}

$$
\begin{aligned}
& a_{4,0}=\theta_{q^{2}}(q)^{2} \theta_{q^{4}}\left(q^{2}\right)+q \theta_{q^{2}}(1)^{2} \theta_{q^{4}}(1), \\
& a_{4,1}=2 \theta_{q^{2}}(1) \theta_{q^{2}}(q) \theta_{q^{4}}\left(q^{3}\right), \\
& a_{4,2}=\theta_{q^{2}}(q)^{2} \theta_{q^{4}}(1)+\theta_{q^{2}}(1)^{2} \theta_{q^{4}}\left(q^{2}\right), \\
& a_{4,3}=2 \theta_{q^{2}}(1) \theta_{q^{2}}(q) \theta_{q^{4}}(q) .
\end{aligned}
$$

Proof. We square (5):

$$
\theta_{q}^{4}(x)=\left(\theta_{q^{2}}(q)\right)^{2}\left(\theta_{q^{2}}\left(x^{2}\right)\right)^{2}+x^{2}\left(\theta_{q^{2}}(1)\right)^{2}\left(\theta_{q^{2}}\left(q x^{2}\right)\right)^{2}+2 x \theta_{q^{2}}(1) \theta_{q^{2}}(q) \theta_{q^{2}}\left(x^{2}\right) \theta_{q^{2}}\left(q x^{2}\right) .
$$

First term, directly from (5) (replacing $q$ by $q^{2}$ and $x$ by $x^{2}$ ):

$$
\left(\theta_{q^{2}}\left(x^{2}\right)\right)^{2}=\theta_{q^{4}}\left(q^{2}\right) \theta_{q^{4}}\left(x^{4}\right)+x^{2} \theta_{q^{4}}(1) \theta_{q^{4}}\left(q^{2} x^{4}\right)
$$

Second term, directly from (5) (replacing $q$ by $q^{2}$ and $x$ by $q x^{2}$ ):

$$
\left(\theta_{q^{2}}\left(q x^{2}\right)\right)^{2}=\theta_{q^{4}}\left(q^{2}\right) \theta_{q^{4}}\left(q^{2} x^{4}\right)+q x^{2} \theta_{q^{4}}(1) \theta_{q^{4}}\left(q^{4} x^{4}\right)=\theta_{q^{4}}\left(q^{2}\right) \theta_{q^{4}}\left(q^{2} x^{4}\right)+q x^{-2} \theta_{q^{4}}(1) \theta_{q^{4}}\left(x^{4}\right)
$$

Third term, appealling to Entry 29 with $x \leftarrow x^{2}$ and $y \leftarrow q x^{2}$ (mean of both items):

$$
\theta_{q^{2}}\left(x^{2}\right) \theta_{q^{2}}\left(q x^{2}\right)=\theta_{q^{4}}\left(q^{3}\right) \theta_{q^{4}}\left(q x^{4}\right)+x^{2} \theta_{q^{4}}(q) \theta_{q^{4}}\left(q^{3} x^{4}\right) .
$$

Collecting:

$$
\begin{aligned}
\theta_{q}^{4}(x) & =\left(\theta_{q^{2}}(q)\right)^{2}\left(\theta_{q^{4}}\left(q^{2}\right) \theta_{q^{4}}\left(x^{4}\right)+x^{2} \theta_{q^{4}}(1) \theta_{q^{4}}\left(q^{2} x^{4}\right)\right) \\
& +x^{2}\left(\theta_{q^{2}}(1)\right)^{2}\left(\theta_{q^{4}}\left(q^{2}\right) \theta_{q^{4}}\left(q^{2} x^{4}\right)+q x^{-2} \theta_{q^{4}}(1) \theta_{q^{4}}\left(x^{4}\right)\right) \\
& +2 x \theta_{q^{2}}(1) \theta_{q^{2}}(q)\left(\theta_{q^{4}}\left(q^{3}\right) \theta_{q^{4}}\left(q x^{4}\right)+x^{2} \theta_{q^{4}}(q) \theta_{q^{4}}\left(q^{3} x^{4}\right)\right) \\
& =\left(\theta_{q^{2}}(q)^{2} \theta_{q^{4}}\left(q^{2}\right)+q \theta_{q^{2}}(1)^{2} \theta_{q^{4}}(1)\right) \theta_{q^{4}}\left(x^{4}\right) \\
& +2 \theta_{q^{2}}(1) \theta_{q^{2}}(q) \theta_{q^{4}}\left(q^{3}\right) x \theta_{q^{4}}\left(q x^{4}\right) \\
& +\left(\theta_{q^{2}}(q)^{2} \theta_{q^{4}}(1)+\theta_{q^{2}}(1)^{2} \theta_{q^{4}}\left(q^{2}\right)\right) x^{2} \theta_{q^{4}}\left(q^{2} x^{4}\right) \\
& +2 \theta_{q^{2}}(1) \theta_{q^{2}}(q) \theta_{q^{4}}(q) x^{3} \theta_{q^{4}}\left(q^{3} x^{4}\right) .
\end{aligned}
$$


Note that, since $\theta_{q^{4}}\left(q^{3}\right)=\theta_{q^{4}}(q)$, we have $a_{4,1}=a_{4,3}$ as was to be expected by (9). Also note that various transformations are possible; for instance, using Entry 18 one can check that

$$
a_{4,1}=a_{4,3}=\frac{1}{2} \theta_{q}(1)^{3} .
$$

In all cases, these coefficients vanish for no value of $q$. However, corollary 2 in 3.1 predicts that $a_{4,0}$ and $a_{4,3}$ vanish for at least one $q<0$ (but says nothing about $a_{4,1}$ and $a_{4,3}$ ).

The process can be (partially) generalized by squaring identity (6). By definition:

$$
\begin{aligned}
\theta_{q}^{2 k}(x) & =\left(\sum_{i=0}^{k-1} a_{k, i} x^{i} \theta_{q^{k}}\left(q^{i} x^{k}\right)\right)^{2} \\
& =\sum_{i, j=0}^{k-1} a_{k, i} a_{k, j} x^{i+j} \theta_{q^{k}}\left(q^{i} x^{k}\right) \theta_{q^{k}}\left(q^{j} x^{k}\right) \\
& =\sum_{i, j=0}^{k-1} a_{k, i} a_{k, j} x^{i+j}\left(\theta_{q^{2 k}}\left(q^{i+j} x^{2 k}\right) \theta_{q^{2 k}}\left(q^{j-i+k}\right)+q^{i} x^{k} \theta_{q^{2 k}}\left(q^{i+j+k} x^{2 k}\right) \theta_{q^{2 k}}\left(q^{j-i}\right)\right) .
\end{aligned}
$$

We used again consequence $\theta_{q}(x) \theta_{q}(y)=\theta_{q^{2}}(x y) \theta_{q^{2}}(q y / x)+x \theta_{q^{2}}(q x y) \theta_{q^{2}}(y / x)$ of Entry 29. Replacing each term $\theta_{q^{2 k}}\left(q^{m} y\right)$ such that $m \geq 2 k$ by $y^{-1} \theta_{q^{2 k}}\left(q^{m-2 k} y\right)$ and collecting carefully, one finds, for $l=0, \ldots, 2 k-1$ :

$$
a_{2 k, l}=\sum_{i+j=l} a_{k, i} a_{k, j} \theta_{q^{2 k}}\left(q^{k+j-i}\right)+\left\{\begin{array}{l}
\sum_{i+j+k=l} a_{k, i} a_{k, j} q^{k-j} \theta_{q^{2 k}}\left(q^{j-i}\right) \text { if } l \leq k-2, \\
\sum_{i+j+k=l} a_{k, i} a_{k, j} q^{i} \theta_{q^{2 k}}\left(q^{j-i}\right) \text { if } l \geq k .
\end{array}\right.
$$

(There is no second term $\sum$ if $l=k-1$.) Taking $k=2$ and using the values of $a_{2,0}$, $a_{2,1}$ given in 1.3, one does recognize the $a_{4, i}$ of corollary 1, Iterative application of these formulas would allow us to determine the $a_{k, i}$ when $k$ is a power of 2 ; but even so, without giving a closed formula. The methods of 2.2 will prove more to be effective.

\subsection{A calculus of coefficients of powers of theta}

\subsubsection{Notations and conventions for this subsection}

We suppose chosen $\tau \in \mathbf{C}$ such that $q=e^{2 \pi i \tau}$ and $\operatorname{Im} \tau>0$, which in particular gives an inambiguous meaning to non integral powers of $q$. In this subsection, in order to have simpler formulas, we rather consider the following theta function:

$$
\vartheta(x):=\vartheta_{q}(x):=\sum_{n \in \mathbf{Z}} q^{n^{2} / 2} x^{n}=\theta_{q}\left(q^{1 / 2} x\right) .
$$

The series $\vartheta$ plainly represents an analytic function all over the punctured complex plane. Let $k \in \mathbf{N}^{*}$ and $n \in \mathbf{Z}$. We write $c_{k, n}(q)$ the coefficient of $x^{n}$ in the Laurent series expansion 
of $\vartheta^{k}(x)$; that is:

$$
c_{k, n}(q)=\sum_{\substack{\left(n_{1}, \ldots, n_{k}\right) \in \mathbf{Z}^{k} \\ n_{1}+\cdots+n_{k}=n}} q^{\left(n_{1}^{2}+\cdots+n_{k}^{2}\right) / 2}
$$

We therefore have:

$$
c_{k, n}=q^{n / 2} \gamma_{k, n}
$$

Setting $n:=0$ in formula (10), we get:

$$
c_{k, 0}(q)=1+k(k-1) q+O\left(q^{2}\right),
$$

where the constant term corresponds to $(0, \ldots, 0)$ and where the coefficient $k(k-1)$ before $q$ comes from the fact that $k$-uples $\left(n_{1}, \ldots, n_{k}\right)$ such that $n_{1}^{2}+\cdots+n_{k}^{2}=2, n_{1}+\cdots+n_{k}=0$ are made of $(k-2)$ zeroes, one +1 and one -1 . More generally, after (8) page 5 ;

$$
c_{k, n}(q)=q^{n / 2}\left(\left(\begin{array}{l}
k \\
n
\end{array}\right)+O(q)\right)
$$

Actually, $\gamma_{k, n}(q)=q^{-n / 2} c_{k, n}(q)$ represents an analytic function over the unit disk, with value $\left(\begin{array}{l}k \\ n\end{array}\right)$ at 0 and its Taylor series at 0 has non negative integer coefficients.

\section{Some notations.}

1. If $f=\sum_{n \in \mathbf{Z}} a_{n} x^{n} \in \mathbf{C}\left[\left[x, \frac{1}{x}\right]\right]$, one puts:

$$
\mathcal{B} f(x):=\mathcal{B}_{q} f(x)=\sum_{n \in \mathbf{Z}} a_{n} q^{n^{2} / 2} x^{n} \in \mathbf{C}\left[\left[x, \frac{1}{x}\right]\right] .
$$

2. If $\mathcal{B}_{q} f$ represents a holomorphic function over $\mathbf{C}^{*}$, one puts:

$$
\mathcal{T} f(x)=\mathcal{T}_{q} f(x)=\sum_{n \in \mathbf{Z}} \mathcal{B}_{q} f\left(q^{-n}\right) x^{n} \in \mathbf{C}\left[\left[x, \frac{1}{x}\right]\right] .
$$

3. For all $a \in \mathbf{C}^{*}$ and $k \in \mathbf{N}^{*}$, one denotes $\sigma_{a} f(x):=f(a x)$ and $\rho_{k} f(x):=f\left(x^{k}\right)$.

4. For $\left(a_{1}, \ldots, a_{m}\right) \in \mathbf{C}^{m}$, one puts:

$$
\left(a_{1}, \ldots, a_{m} ; q\right)_{\infty}:=\prod_{n=1}^{m}\left(a_{n} ; q\right)_{\infty} .
$$

(Pochhammer symbols $(a ; q)_{\infty}$ were defined at the beginning of 2.1.1.) 
Remark 1. We here recall some useful facts:

1. $\vartheta(1 / x)=\vartheta(x) ; \vartheta\left(q^{n} x\right)=q^{-n^{2} / 2} x^{-n} \vartheta(x)$ for $n \in \mathbf{Z}$.

2. $\vartheta(x)=\left(q,-\sqrt{q} x,-\frac{\sqrt{q}}{x} ; q\right)_{\infty}$.

3. $\vartheta(x)=0$ if, and only if $x \in-q^{\mathbf{Z}+\frac{1}{2}}$.

4. $\prod_{n=0}^{k-1} \vartheta_{q^{k}}\left(x q^{n}\right)=\frac{\left(q^{k} ; q^{k}\right)_{\infty}^{k}}{(q ; q)_{\infty}} \vartheta\left(x q^{(k-1) / 2}\right)$ for $k \in \mathbf{N}^{*}$.

5. $\mathcal{B}_{q^{k}} \vartheta_{q^{m}}=\vartheta_{q^{k+m}}$ for $k, m \in \mathbf{N}^{*}$.

6. $\mathcal{B}\left(x^{n} f\right)=q^{n^{2} / 2} x^{n} \mathcal{B} f$ for $n \in \mathbf{Z}$.

7. $\sigma_{a} \mathcal{B}=\mathcal{B} \sigma_{a}$ for $a \in \mathbf{C}^{*}$.

8. $\mathcal{B}_{q} \rho_{k}=\rho_{k} \mathcal{B}_{q^{k^{2}}}$ for $k \in \mathbf{N}^{*}$.

Proposition 2. If $\mathcal{B}_{q} f$ represents a holomorphic function over $\mathbf{C}^{*}$, one has: $\vartheta f=\mathcal{B}(\mathcal{T} f)$.

Proof. Immediate calculation.

For $f=\vartheta$, remark 1 (5) says that $\mathcal{B} \vartheta=\vartheta_{q^{2}}$, which implies:

$$
\mathcal{T} \vartheta(x)=\sum_{n \in \mathbf{Z}} \vartheta_{q^{2}}\left(q^{-n}\right) x^{n}=\sum_{n \in \mathbf{Z}} \vartheta_{q^{2}}\left(q^{n}\right) x^{n}
$$

With the help of proposition 2, one finds the following expression:

$$
\vartheta^{2}(x)=\sum_{n \in \mathbf{Z}} \vartheta_{q^{2}}\left(q^{n}\right) q^{n^{2} / 2} x^{n}
$$

According to remark 1 (3), we know that $\vartheta_{q^{2}}\left(q^{n}\right) \neq 0$ except if $q^{n} \in-q^{2 \mathbf{Z}+1}$, which is impossible since- $1 \notin q^{\mathbf{Z}}$. Therefore:

Fact. The series expanding $\vartheta^{2}$ has no null coefficient.

In order to go on, let us introduce some more general notations. Let $k \in \mathbf{N}^{*}$. Replacing $n$ by 1 and then $\vartheta$ by $\vartheta^{k}$ in the second functional equation of remark 1 (1), we find that $\sigma_{q} \vartheta^{k}(x)=q^{-k / 2} x^{-k} \vartheta^{k}(x)$. Moreover, if $T_{k, n}(x)=x^{n} \vartheta_{q^{k}}\left(x^{k} q^{n}\right)$ with $n \in \mathbf{Z}$, one has $\sigma_{q} T_{k, n}(x)=q^{-k / 2} x^{-k} T_{k, n}(x)$. This being said, the functions $T_{k, n}$ all satisfy the same linear equation as $\vartheta^{k}$ (compare 1.3.1). 


\subsubsection{A recurrence relation}

Proposition 3. For every $k \in \mathbf{N}^{*}$, we have:

$$
\vartheta^{k}(x)=\sum_{n=0}^{k-1} c_{k, n}(q) x^{n} \vartheta_{q^{k}}\left(x^{k} q^{n}\right)
$$

Moreover, the coefficients $c_{k, n}(q)$ satisfy the following recurrence relation:

$$
c_{k+1, n}(q)=\sum_{n^{\prime}=0}^{k-1} c_{k, n^{\prime}}(q) q^{\left(n-n^{\prime}\right)^{2} / 2} \vartheta_{q^{k^{2}+k}}\left(q^{-k n+(k+1) n^{\prime}}\right) .
$$

Proof. We shall apply proposition 2 to a function of the form $f(x)=\vartheta_{q^{k}}\left(x^{k} q^{n}\right)$, where $k \in \mathbf{N}^{*}$ et $n \in\{0, \ldots, k-1\}$. Write $\vartheta_{q^{k}}\left(x^{k} q^{n}\right)=\sigma_{q^{n / k}} \rho_{k} \vartheta_{q^{k}}(x)$ and use remark11. We have:

$$
\mathcal{B} \vartheta_{q^{k}}\left(x^{k} q^{n}\right)=\mathcal{B} \sigma_{q^{n / k}} \rho_{k} \vartheta_{q^{k}}(x)=\cdots=\vartheta_{q^{k^{2}+k}}\left(x^{k} q^{n}\right) \text {. }
$$

We deduce that:

$$
\vartheta(x) \vartheta_{q^{k}}\left(x^{k} q^{n}\right)=\mathcal{B}(\mathcal{T} f)(x)=\sum_{m \in \mathbf{Z}} \vartheta_{q^{k^{2}+k}}\left(q^{-m k+n}\right) q^{m^{2} / 2} x^{n}
$$

If $m=(k+1) v+r$ with $v \in \mathbf{Z}$ and $r \in\{-n, 1-n, \ldots, k-n\}$, we write $q^{-m k+n}=q^{-(k+1) k v-k r+n}$ and get:

$$
\vartheta_{q^{k^{2}+k}}\left(q^{-m k+n}\right)=q^{-k(k+1) v^{2} / 2} q^{(-k r+n) v} \vartheta_{q^{k^{2}+k}}\left(q^{-k r+n}\right) .
$$

With $m^{2}=(k+1)^{2} v^{2}+2(k+1) r v+r^{2}$, it follows that:

$$
\vartheta_{q^{k^{2}+k}}\left(q^{-m k+n}\right) q^{m^{2} / 2}=q^{(k+1) v^{2} / 2} q^{(r+n) v} q^{r^{2} / 2} \vartheta_{q^{k^{2}+k}}\left(q^{-k r+n}\right) .
$$

Hence expression (17) becomes:

$$
\vartheta(x) \vartheta_{q^{k}}\left(x^{k} q^{n}\right)=\sum_{r=-n}^{k-n} q^{r^{2} / 2} \vartheta_{q^{k^{2}+k}}\left(q^{-k r+n}\right) x^{r} \vartheta_{q^{k+1}}\left(x q^{r+n}\right)
$$

Using (15), we deduce that (18):

$$
\vartheta^{k+1}(x)=\sum_{r=-n}^{k-n} q^{r^{2} / 2} \sum_{n=0}^{k-1} c_{k, n}(q) \vartheta_{q^{k^{2}+k}}\left(q^{-k r+n}\right) x^{r+n} \vartheta_{q^{k+1}}\left(x q^{r+n}\right) .
$$

This completes the proof of the recurrence relation (16). In this way, we finish the proof of proposition 3 . 


\subsubsection{A symmetry relation}

As already noted in 1.3.2, equation (9), $\gamma_{k, n}=\gamma_{k, k-n}$. Therefore, iff $0<n<k$, one has the following symmetry:

$$
c_{k, n}(q)=q^{n-k / 2} c_{k, k-n}(q) .
$$

Let us explain how to use (19) so as to shorten the expression of recurrence (16). First assume that $k$ is odd $\geq 1$, and write $k=2 k^{\prime}+1$. Grouping the terms in the sum from (16) according to whether $n^{\prime}=0$ or $1 \leq n^{\prime} \leq k^{\prime}$ or $n^{\prime}>k^{\prime}$, one has:

(20)

$$
\begin{gathered}
c_{k+1, n}(q)=c_{k, 0}(q) q^{n^{2} / 2} \vartheta_{q^{k^{2}+k}}\left(q^{-k n}\right)+\sum_{n^{\prime}=1}^{k^{\prime}}\left(c_{k, n^{\prime}}(q) q^{\left(n-n^{\prime}\right)^{2} / 2} \vartheta_{q^{k^{2}+k}}\left(q^{-k n+(k+1) n^{\prime}}\right)\right. \\
\left.+c_{k, k^{\prime}+n^{\prime}}(q) q^{\left(n-k^{\prime}-n^{\prime}\right)^{2} / 2} \vartheta_{q^{k^{2}+k}}\left(q^{-k n+(k+1)\left(k^{\prime}+n^{\prime}\right)}\right)\right) .
\end{gathered}
$$

From (19), one has: $c_{k, k^{\prime}+n^{\prime}}(q)=q^{n^{\prime}-1 / 2} c_{k, k^{\prime}+1-n^{\prime}}$. Writing $k^{\prime}+n^{\prime}=k-1-k^{\prime}+n^{\prime}$, we draw $(k+1)\left(k^{\prime}+n^{\prime}\right)=(k+1) k+(k+1)\left(n^{\prime}-k^{\prime}-1\right)$, which yields:

$$
\begin{aligned}
\vartheta_{q^{k^{2}+k}}\left(q^{-k n+(k+1)\left(k^{\prime}+n^{\prime}\right)}\right) & =\vartheta_{q^{k^{2}+k}}\left(q^{-k n+(k+1)\left(n^{\prime}-k^{\prime}-1\right)+(k+1) k}\right) \\
& =q^{k n-(k+1)\left(n^{\prime}-1 / 2\right)} \vartheta_{q^{k^{2}+k}}\left(q^{-k n+(k+1)\left(n^{\prime}-k^{\prime}-1\right)}\right) .
\end{aligned}
$$

Since $k n-(k+1)\left(n^{\prime}-1 / 2\right)=\left(2 k^{\prime}+1\right)\left(n-n^{\prime}\right)+k^{\prime}+1-n^{\prime}$, one has:

$$
q^{n^{\prime}-1 / 2} q^{\left(n-k^{\prime}-n^{\prime}\right)^{2} / 2} q^{k n-(k+1)\left(n^{\prime}-1 / 2\right)}=q^{\left(n-n^{\prime}+k^{\prime}+1\right)^{2} / 2} .
$$

As a consequence, if $n^{\prime \prime}=k^{\prime}+1-n^{\prime}$, one finds:

$$
\begin{aligned}
c_{k, k^{\prime}+n^{\prime}}(q) q^{\left(n-k^{\prime}-n^{\prime}\right)^{2} / 2} & \vartheta_{q^{k^{2}+k}}\left(q^{-k n+(k+1)\left(k^{\prime}+n^{\prime}\right)}\right) \\
& =c_{k, n^{\prime \prime}} q^{\left(n+n^{\prime \prime}\right)^{2} / 2} \vartheta_{q^{k^{2}+k}}\left(q^{-k n-(k+1) n^{\prime \prime}}\right) .
\end{aligned}
$$

When $n^{\prime}$ goes from 1 to $k^{\prime}, n^{\prime \prime}$ runs along the same values in descending order. Going back to formula (20), one is led to the following remark.

Fact. If $k=2 k^{\prime}+1$ and $k^{\prime} \in \mathbf{N}$, then:

$$
\begin{aligned}
c_{k+1, n}(q)= & c_{k, 0}(q) q^{n^{2} / 2} \vartheta_{q^{k^{2}+k}}\left(q^{k n}\right)+\sum_{n^{\prime}=1}^{k^{\prime}} c_{k, n^{\prime}}(q) \times \\
& \left(q^{\left(n^{\prime}-n\right)^{2} / 2} \vartheta_{q^{k^{2}+k}}\left(q^{k\left(n^{\prime}-n\right)+n^{\prime}}\right)+q^{\left(n^{\prime}+n\right)^{2} / 2} \vartheta_{q^{k^{2}+k}}\left(q^{k\left(n^{\prime}+n\right)+n^{\prime}}\right)\right) .
\end{aligned}
$$

In particular, for $n=0$, one has:

$$
c_{k+1,0}(q)=c_{k, 0}(q) \vartheta_{q^{k^{2}+k}}(1)+2 \sum_{n=1}^{k^{\prime}} c_{k, n}(q) q^{n^{2} / 2} \vartheta_{q^{k^{2}+k}}\left(q^{(k+1) n}\right) .
$$


Consider now the case when $k=2 k^{\prime}$ with $k^{\prime} \in \mathbf{N}^{*}$. Using relation (16), one can check that

$$
\begin{gathered}
c_{k+1, n}(q)=c_{k, 0}(q) q^{n^{2} / 2} \vartheta_{q^{k^{2}+k}}\left(q^{-k n}\right)+c_{k, k^{\prime}}(q) q^{\left(n-k^{\prime}\right)^{2} / 2} \vartheta_{q^{k^{2}+k}}\left(q^{-k n+(k+1) k^{\prime}}\right) \\
+\sum_{n^{\prime}=1}^{k^{\prime}-1}\left(c_{k, n^{\prime}}(q) q^{\left(n-n^{\prime}\right)^{2} / 2} \vartheta_{q^{k^{2}+k}}\left(q^{-k n+(k+1) n^{\prime}}\right)\right. \\
\left.\quad+c_{k, k^{\prime}+n^{\prime}}(q) q^{\left(n-k^{\prime}-n^{\prime}\right)^{2} / 2} \vartheta_{q^{k^{2}+k}}\left(q^{-k n+(k+1)\left(k^{\prime}+n^{\prime}\right)}\right)\right) .
\end{gathered}
$$

A similar analysis would entail the following remark.

Fact. If $k=2 k^{\prime}$ et $k^{\prime} \in \mathbf{N}^{*}$, then:

$$
\begin{gathered}
c_{k+1, n}(q)=c_{k, 0}(q) q^{n^{2} / 2} \vartheta_{q^{k^{2}+k}}\left(q^{k n}\right)+c_{k, k^{\prime}}(q) q^{\left(n-k^{\prime}\right)^{2} / 2} \vartheta_{q^{k^{2}+k}}\left(q^{k\left(k^{\prime}-n\right)+k^{\prime}}\right) \\
+\sum_{n^{\prime}=1}^{k^{\prime}-1} c_{k, n^{\prime}}(q) \\
\left(q^{\left(n^{\prime}-n\right)^{2} / 2} \vartheta_{q^{k^{2}+k}}\left(q^{k\left(n^{\prime}-n\right)+n^{\prime}}\right)\right. \\
\left.+q^{\left(n^{\prime}+n\right)^{2} / 2} \vartheta_{q^{k^{2}+k}}\left(q^{k\left(n^{\prime}+n\right)+n^{\prime}}\right)\right) .
\end{gathered}
$$

In particular, for $n=0$, one has:

$$
\begin{aligned}
c_{k+1,0}(q)= & c_{k, 0}(q) \vartheta_{q^{k^{2}+k}}(1)+c_{k, k^{\prime}}(q) q^{k^{2} / 8} \vartheta_{q^{k^{2}+k}}\left(q^{(k+1) k / 2}\right) \\
& +2 \sum_{n=1}^{k^{\prime}-1} c_{k, n}(q) q^{n^{2} / 2} \vartheta_{q^{k^{2}+k}}\left(q^{(k+1) n}\right) .
\end{aligned}
$$

\subsubsection{About coefficients $c_{k, n}(q)$ for $k=2$ or 3}

Putting $k:=1$ in (16), since $c_{1,0}(q)=1$, we find:

$$
c_{2, n}(q)=\vartheta_{q^{2}}\left(q^{n}\right) q^{n^{2} / 2}, \quad n \in\{0,1\} .
$$

Moreover, with $k=2$ and $\mu=-1$ in (43), we have:

$$
c_{2, n}(q)=\frac{q^{n^{2} / 2} x^{-n}}{2 \vartheta_{q^{2}}\left(x^{2}\right)}\left(\vartheta^{2}\left(x q^{-n / 2}\right)+(-1)^{n} \vartheta^{2}\left(-x q^{-n / 2}\right)\right) .
$$

Let us set $x=q^{(n+1) / 2}$, and recall that $\vartheta(-\sqrt{q})=0$; one deduces the expression $c_{2, n}(q)=$ $\frac{q^{-n / 2} \vartheta^{2}(\sqrt{q})}{2 \vartheta_{q^{2}}\left(q^{n+1}\right)}$. Comparing the latter with $(26)$ for $n=0$ gives the following identity:

$$
\vartheta^{2}(\sqrt{q})=2 \vartheta_{q^{2}}(1) \vartheta_{q^{2}}(q)
$$


Applying relation 14 (4) for $k=2$ and $x=1$, one has:

$$
\vartheta_{q^{2}}(1) \vartheta_{q^{2}}(q)=\frac{\left(q^{2} ; q^{2}\right)_{\infty}^{2}}{(q ; q)_{\infty}} \vartheta(\sqrt{q})=\frac{\left(q^{2} ; q^{2}\right)_{\infty}^{2}}{(q ; q)_{\infty}} \vartheta(\sqrt{q}) .
$$

Identity (27) can then be read as follows:

$$
\vartheta(\sqrt{q})=2 \frac{\left(q^{2} ; q^{2}\right)_{\infty}^{2}}{(q ; q)_{\infty}}
$$

where the factor 2 looks almost unexpected ...

Let us compute $c_{3, n}(q)$ for $n=0,1,2$ with the help of (16):

$$
c_{3, n}(q)=c_{2,0}(q) q^{n^{2} / 2} \vartheta_{q^{6}}\left(q^{2 n}\right)+c_{2,1}(q) q^{(n-1)^{2} / 2} \vartheta_{q^{6}}\left(q^{2 n-3}\right) .
$$

With (26), one finds:

$$
c_{3, n}(q)=q^{n^{2} / 2} \vartheta_{q^{2}}(1) \vartheta_{q^{6}}\left(q^{2 n}\right)+q^{\left(1+(n-1)^{2}\right) / 2} \vartheta_{q^{2}}(q) \vartheta_{q^{6}}\left(q^{2 n-3}\right) .
$$

In particular, one notes that:

$$
c_{3,0}(q)=\vartheta_{q^{2}}(1) \vartheta_{q^{6}}(1)+q \vartheta_{q^{2}}(q) \vartheta_{q^{6}}\left(q^{3}\right) .
$$

If one puts $f(q)=\vartheta_{q^{2}}(1)$ and $g(q)=\vartheta_{q^{2}}(q)$, one has:

$$
c_{3,0}(q)=f(q) f\left(q^{3}\right)+q g(q) g\left(q^{3}\right) .
$$

The functions $f$ and $g$ may be written in the following form:

$$
f(q)=1+2 \sum_{n \geq 1} q^{n^{2}}, \quad g(q)=2+2 \sum_{n \geq 1} q^{n(n+1)},
$$

where the power series all have the unit circle as convergence boundary. If $q \rightarrow-1^{-}$, one sees that $|f(q)|<1$ but $g(q) \rightarrow+\infty$. Considering expression (30), one draws that $c_{3,0}(q) \rightarrow-\infty$ for $q \rightarrow-1^{-}$. Since $c_{3,0}(0)=1$, one infers:

Fact. $c_{3,0}(q)$ admits (at least) a zero over $]-1,0[$.

Using Maple, by chosing values of $n$ up to $N=600$, one more or less gets the graph of $q \mapsto c_{3,0}(q)$ over interval ] $-0.5,0.5$ [. Depending on the look of the graph, one can ask Maple to evaluate $c_{3,0}(q)$ for $a=-0.163034$ and $b=-0.163033$. Herebelow some of the numerical values.

For $N=400$ :

$$
c_{3,0}(a)=-2.96590 * 10^{-6}, \quad c_{3,0}(b)=3.41022854 * 10^{-6} .
$$

For $N=500$ :

$$
c_{3,0}(a)=-2.96589725 * 10^{-6}, \quad c_{3,0}(b)=3.41023 * 10^{-6} .
$$

For $N=600$ :

$$
c_{3,0}(a)=-2.96589725 * 10^{-6}, \quad c_{3,0}(b)=3.41022854 * 10^{-6} .
$$


Remark 2. It seems that $c_{3,0}(q)$ vanishes somewhere between $a=-0.163034$ and $b=$ -0.163033 .

Put $n=1$ in (29); then:

$$
c_{3,1}(q)=q^{1 / 2}\left(\vartheta_{q^{2}}(1) \vartheta_{q^{6}}\left(q^{2}\right)+\vartheta_{q^{2}}(q) \vartheta_{q^{6}}(q)\right) .
$$

We find likewise:

$$
c_{3,2}(q)=q\left(q \vartheta_{q^{2}}(1) \vartheta_{q^{6}}\left(q^{4}\right)+\vartheta_{q^{2}}(q) \vartheta_{q^{6}}(q)\right)
$$

Noting that $\vartheta_{q^{6}}\left(q^{4}\right)=\vartheta_{q^{6}}\left(q^{-4}\right)=q^{-1} \vartheta_{q^{6}}\left(q^{2}\right)$, we get:

$$
c_{3,2}(q)=q^{1 / 2} c_{3,1}(q) .
$$

This is consistent with the symmetry stated in (19).

\subsubsection{About coefficients $c_{4, n}(q)$}

Putting $k^{\prime}=1$ and $k=3$ in (21), we obtain that:

$$
\begin{aligned}
c_{4, n}(q)= & c_{3,0}(q) q^{n^{2} / 2} \vartheta_{q^{12}}\left(q^{k n}\right)+c_{3,1}(q) \times \\
& \left(q^{(n-1)^{2} / 2} \vartheta_{q^{12}}\left(q^{-3 n+4}\right)+q^{(n+1)^{2} / 2} \vartheta_{q^{12}}\left(q^{3 n+4}\right)\right) .
\end{aligned}
$$

For $n=0$, formula (22) implies:

$$
c_{4,0}(q)=c_{3,0}(q) \vartheta_{q^{12}}(1)+2 c_{3,1}(q) q^{1 / 2} \vartheta_{q^{12}}\left(q^{4}\right) .
$$

In account of (29), one can check that:

$$
\begin{aligned}
c_{4,0}(q)= & \vartheta_{q^{2}}(1) \vartheta_{q^{6}}(1) \vartheta_{q^{12}}(1)+q\left[\vartheta_{q^{2}}(q) \vartheta_{q^{6}}\left(q^{3}\right) \vartheta_{q^{12}}(1)\right. \\
& \left.+2\left(\vartheta_{q^{2}}(1) \vartheta_{q^{6}}\left(q^{2}\right)+\vartheta_{q^{2}}(q) \vartheta_{q^{6}}(q)\right) \vartheta_{q^{12}}\left(q^{4}\right)\right] .
\end{aligned}
$$

It may be noted that both (33) and (34) are much more complicated that the formulas found in Corollary 1.

\section{Asymptotics of the powers of theta and vanishing of the coefficients}

\subsection{Some sufficient conditions for the vanishing of $\gamma_{k, n}(q)$}

\subsubsection{Main statement and reduction to an asymptotic estimation}

Recall that, for $k \geq 1$, underlined characters denote $k$-uples: $\underline{x}=\left(x_{1}, \ldots, x_{k}\right)$. 
We first introduce a linear and a quadratic form on $\mathbf{R}^{k}$ :

$$
S(\underline{x}):=\sum_{1 \leq i \leq k} x_{i}, \quad Q(\underline{x}):=\sum_{1 \leq i \leq j \leq k} x_{i} x_{j}=\frac{1}{2}\left(\sum_{1 \leq i \leq k} x_{i}^{2}+S(\underline{x})^{2}\right) .
$$

The last equality shows that $Q$ is a positive definite form.

Lemma 1. Let $k \in \mathbf{N}^{*}$ and $n \in \mathbf{Z}$. Put, for $x \in \mathbf{C},|x|<1$ :

$$
f_{k, n}(x):=\sum_{\underline{m} \in \mathbf{Z}^{k}} x^{Q(\underline{m})-n S(\underline{m})} .
$$

Then:

$$
\forall q \in \mathbf{C} \backslash \overline{\mathbf{D}}(0,1), \gamma_{k+1, n}(q)=q^{n(n-1) / 2} f_{k, n}(q)
$$

The function $f_{k, n}$ is holomorphic over the punctured open disk $\stackrel{\circ}{\mathbf{D}}(0,1) \backslash\{0\}$ with a pole of order $n(n-1) / 2$ at 0 (meaning that if $n \in\{0,1\}$ then $f_{k, n}$ is actually holomorphic at 0 ).

Proof. From formula (2) one draws the following calculation:

$$
\begin{aligned}
\gamma_{k+1, n}(q) & =\sum_{\substack{m_{0}, \ldots, m_{k} \in \mathbf{Z} \\
m_{0}+\cdots+m_{k}=n}} q^{\frac{m_{0}^{2}+\cdots+m_{k}^{2}-m_{0}-\cdots-m_{k}}{2}} \\
& =\sum_{m_{1}, \ldots, m_{k} \in \mathbf{Z}} q^{\frac{\left(m_{1}+\cdots+m_{k}-n\right)^{2}+m_{1}^{2}+\cdots+m_{k}^{2}-n}{2}} \\
& =\sum_{m_{1}, \ldots, m_{k} \in \mathbf{Z}} q^{\frac{\left(m_{1}+\cdots+m_{k}\right)^{2}-2 n\left(m_{1}+\cdots+m_{k}\right)+n^{2}+m_{1}^{2}+\cdots+m_{k}^{2}-n}{2}} \\
& =q^{n(n-1) / 2} \sum_{m_{1}, \ldots, m_{k} \in \mathbf{Z}} q^{\frac{\left(m_{1}+\cdots+m_{k}\right)^{2}-2 n\left(m_{1}+\cdots+m_{k}\right)+m_{1}^{2}+\cdots+m_{k}^{2}}{2}} \\
& =q^{n(n-1) / 2} \sum_{\underline{m} \in \mathbf{Z}^{k}} q^{Q(\underline{m})-n S(\underline{m})} .
\end{aligned}
$$

Theorem 1. Let $k \in \mathbf{N}^{*}$ and $n \in \mathbf{Z}$. Writing $\varepsilon:=(-1)^{n}$, one has, when $x \rightarrow 1^{-}$:

$$
f_{k, n}(-x)=\frac{\pi^{\frac{k}{2}} \sqrt{2}}{\sqrt{k+1}} \frac{\cos ((k+\varepsilon) \pi / 4)}{(1-x)^{\frac{k}{2}}}+O\left(\frac{1}{(1-x)^{\frac{k-1}{2}}}\right) .
$$

From 3.1.2 on, most of the rest3 of subsection 3.1 is devoted to the proof of theorem 1

\footnotetext{
${ }^{3}$ Note however that in 3.1 .4 we apply modular properties of generating series of number of representations by quadratic forms to obtain some complementary informations (proposition7).
} 
Consequences of theorem 1. We first discuss here consequences of this theorem of interest for our problem (possibility of vanishing of the coefficients $\gamma_{k, n}$ ). Assume $k \geq 2$ and $0 \leq n \leq k$, so that $\gamma_{k, n}(0)=\left(\begin{array}{l}k \\ n\end{array}\right)>0$ by formula (8) page 5. On the other hand, the sign of $\gamma_{k, n}(q)$ as $q \rightarrow-1$ while staying within $]-1,0\left[\right.$ is (after lemma 35) $(-1)^{n(n-1) / 2} \times$ $\cos k^{\prime} \pi / 4$, where $k^{\prime}:=k-1+(-1)^{n}$; so this sign depends on $k(\bmod 8) \in\{0, \ldots, 7\}$ and on $n(\bmod 4) \in\{0, \ldots, 3\}$ (see our notational conventions at the very end of section 1 ). More precisely:

$$
(-1)^{n(n-1) / 2}=\left\{\begin{array}{l}
+1 \text { if } n(\bmod 4) \in\{0,1\}, \\
-1 \text { if } n(\bmod 4) \in\{2,3\},
\end{array}\right.
$$

and

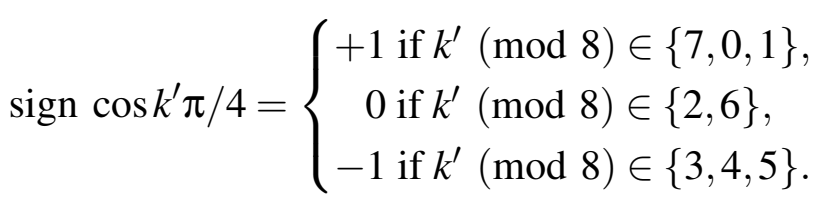

Now, $k^{\prime}=k$ if $n$ is even and $k-2$ if $n$ is odd, so that we list the cases when $\gamma_{k, n}(q)<0$ as $q \rightarrow-1$, we know that in all these cases it must vanish somewhere.

Corollary 2. Assume $k \geq 2$ and $0 \leq n \leq k$. In all the following cases, $\gamma_{k, n}(q)$ vanishes for at least one value of $q \in]-1,0[$ :

- $n(\bmod 4)=0$ and $k(\bmod 8) \in\{3,4,5\}$,

- $n(\bmod 4)=1$ and $k(\bmod 8) \in\{5,6,7\}$,

- $n(\bmod 4)=2$ and $k(\bmod 8) \in\{7,0,1\}$,

- $n(\bmod 4)=3$ and $k(\bmod 8) \in\{1,2,3\}$,

All these conditions (i.e. their logical disjunction) can be summarized in a unique one:

$$
k-2 n(\bmod 8) \in\{3,4,5\} .
$$

Note that the above conditions exclude $a$ priori the case $k=2$ (because for $n=0,1,2$ one does not have $k-2 n(\bmod 8) \in\{3,4,5\})$, so in what follows we assume that $k \geq 3$ and write:

$$
Z_{k}:=\left\{n \in \mathbf{Z} \mid \gamma_{k, n}(q) \text { vanishes somewhere in }\right]-1,0[\} \text {. }
$$

By the remark at the end of 1.3.1, $Z_{k}$ is $k$-periodic i.e. $n \in Z_{k} \Rightarrow n+k \mathbf{Z} \subset Z_{k}$. In order to use that fact, we introduce some more notations:

$$
\begin{aligned}
X_{k} & :=\{n \in\{0, \ldots, k\} \mid k-2 n(\bmod 8) \in\{3,4,5\}\}, \\
Y_{k} & :=X_{k}+k \mathbf{Z} .
\end{aligned}
$$

Note that $X_{k}$ is invariant under the symmetry $n \leftrightarrow k-n$, and therefore so is $Y_{k}$ : we shall not have to exploit further the symmetry expressed by equation (9), it is already built-in. With the previous notations, we thus have:

Corollary 3. Let $k \geq 3$. Then $Y_{k} \subset Z_{k}$.

To make this more useful, we describe more precisely $X_{k}$, and, in some cases, $Y_{k}$. 
Case $k$ even: $k=8 a+2 b, b \in\{0,1,2,3\}$. Then in condition $k-2 n(\bmod 8) \in\{3,4,5\}$ only the remainder 4 is possible:

$$
X_{k}=\{n \in\{0, \ldots, k\} \mid k \equiv 2 n+4 \quad(\bmod 8)\}=\{n \in\{0, \ldots, k\} \mid n \equiv b+2 \quad(\bmod 4)\} .
$$

For instance, one easily checks the following cases:

- If $k=8 a$, then $X_{k}=4\{0, \ldots, 2 a\}+2$ and $Y_{k}=4 \mathbf{Z}+2$.

- If $k=8 a+4$, then $X_{k}=4\{0, \ldots, 2 a\}$ and $Y_{k}=4 \mathbf{Z}$.

Corollary 4. All $\gamma_{8 a, 4 c+2}(q)$ and all $\gamma_{8 a+4,4 c}(q)$ vanish for some $\left.q \in\right]-1,0[$.

Cases $k=8 a+2$ and $k=8 a+6$ can be similarly described (but less simply).

Case $k$ odd: $k=8 a+2 b+1, b \in\{0,1,2,3\}$. Then in condition $k-2 n(\bmod 8) \in$ $\{3,4,5\}$ only cases 3 and $5 \equiv-3$ are possible:

$X_{k}=\{n \in\{0, \ldots, k\} \mid k \equiv 2 n \pm 3 \quad(\bmod 8)\}=\{n \in\{0, \ldots, k\} \mid n \equiv b+2$ or $b-1 \quad(\bmod 4)\}$.

Again these cases can be similarly but less simply described. We just give an example: if $k=3$, we find that $X_{3}=\{0,2\}$ and $Y_{3}=3 \mathbf{Z} \pm 1$.

\subsubsection{Two auxiliary propositions and an application to a generating series}

First auxiliary proposition. Let $L(\underline{y}):=\sum_{1 \leq i \leq k} b_{i} y_{i}$ a linear form and $F(\underline{y}):=\sum_{1 \leq i \leq j \leq k} a_{i, j} y_{i} y_{j}$ a positive definite quadratic form on $\mathbf{R}^{k}$. Write, for $M>0$ :

$$
E_{M}:=\left\{\underline{y} \in \mathbf{R}^{k} \mid F(\underline{y})+L(\underline{y}) \leq M\right\} \quad \text { and } \quad \Lambda_{M}:=E_{M} \cap \mathbf{Z}^{k} .
$$

Since $F$ is positive definite, $E_{M}$ is a compact ellipsoid and $\Lambda_{M}$ is a finite set.

The volume of $E_{M}$ is easily computed as follows. Let $V_{k}$ the volume of the unit ball of $\mathbf{R}^{k}$ and let $D_{F}$ the discriminant of $F$. The matrix of $F$ writes ${ }^{t} S S$, where $S$ is invertible, and $D_{F}=(\operatorname{det} S)^{2}$. Therefore $F(\underline{y})=(S \underline{y})^{2}:=\langle S \underline{y}, S \underline{y}\rangle$. In the same way, $L(\underline{y})=B \underline{y}$ for some line matrix $B$. If $\underline{y}_{0}:=\frac{1}{2} t\left(B S^{-1}\right)$, one sees that $\left\langle S \underline{y}+\underline{y}_{0}, S \underline{y}+\underline{y}_{0}\right\rangle^{2}=F(\underline{y})+L(\underline{y})+\underline{y}_{0}^{2}$. Thus, the mapping $\underline{y} \mapsto S \underline{y}+\underline{y}_{0}$ transforms $E_{M}$ into a ball with radius $\sqrt{M+\underline{y}_{0}^{2}}$, the volume of which is $V_{k}\left(M+\underline{y}_{0}^{2}\right)^{k / 2}$. On the other hand, this affine linear mapping multiplies all volumes by $|\operatorname{det} S|=D_{F}^{1 / 2}$. One concludes:

$$
\mu\left(E_{M}\right)=\frac{V_{k}}{D_{F}^{1 / 2}}\left(M+\underline{y}_{0}^{2}\right)^{k / 2} .
$$


Proposition 4. Let $V_{k}$ the volume of the unit ball of $\mathbf{R}^{k}$ and let $D_{F}$ the discriminant of $F$.

Then, when $M \rightarrow+\infty$ :

$$
\operatorname{card} \Lambda_{M}=\frac{V_{k}}{D_{F}^{1 / 2}} M^{k / 2}+O\left(M^{(k-1) / 2}\right)
$$

Proof. Let $E_{M}^{\prime}:=\Lambda_{M}+[-1 / 2,1 / 2]^{k}$, the volume $\mu\left(E_{M}^{\prime}\right)$ of which is card $\Lambda_{M}$. We shall first determine $M^{\prime}, M^{\prime \prime}>0$ such that $E_{M^{\prime}} \subset E_{M}^{\prime} \subset E_{M^{\prime \prime}}$, which will provide us uper and lower bound for card $\Lambda_{M}=\mu\left(E_{M}^{\prime}\right)$.

Let $\underline{y} \in \Lambda_{M}$ and $\underline{u} \in[-1 / 2,1 / 2]^{k}$ and let us write $\phi:=F+L$. Triangle inequalities applied to the norm $\sqrt{F}$ imply:

$$
\sqrt{F(\underline{y})}-a \leq \sqrt{F(\underline{y})}-\sqrt{F(\underline{u})} \leq \sqrt{F(\underline{y}+u)} \leq \sqrt{F(\underline{y})}+\sqrt{F(\underline{u})} \leq \sqrt{F(\underline{y})}+a,
$$

where $a:=\frac{1}{2} \sqrt{\sum_{1 \leq i \leq j \leq k}\left|a_{i, j}\right|}$, because $F(\underline{u}) \leq a^{2}$ for every $\underline{u} \in[-1 / 2,1 / 2]^{k}$. One deduces:

$$
F(\underline{y})-2 a \sqrt{F(\underline{y})}+a^{2} \leq F(\underline{y}+\underline{u}) \leq F(\underline{y})+2 a \sqrt{F(\underline{y})}+a^{2} .
$$

In the same way, $L(\underline{y})-b \leq L(\underline{y}+\underline{u})=L(\underline{y})+L(\underline{u}) \leq L(\underline{y})+b$, where $b:=\frac{1}{2} \sum_{1 \leq i \leq k}\left|b_{i}\right|$. One draws:

$$
\phi(\underline{y})+a^{2}-b-2 a \sqrt{F(\underline{y})} \leq \phi(\underline{y}+\underline{u}) \leq \phi(\underline{y})+a^{2}+b+2 a \sqrt{F(\underline{y})} .
$$

On the other hand, there exists a constant $c>0$ such that $|L| \leq c \sqrt{F}$ (continuity of the linear form $L$ with respect to the topology defined by the norm $\sqrt{F}$, all this in finite dimension). One deduces:

$$
\phi(\underline{y}) \geq F(\underline{y})-c \sqrt{F(\underline{y})}=(\sqrt{F(\underline{y})}-c / 2)^{2}-c^{2} / 4 \Longrightarrow \sqrt{F(\underline{y})} \leq c / 2+\sqrt{\phi(\underline{y})+c^{2} / 4} .
$$

In the end, we find, for $d^{\prime}:=a^{2}-b-a c$ and $d^{\prime \prime}:=a^{2}+b+a c$, the bounds:

$$
\phi(\underline{y})-2 a \sqrt{\phi(\underline{y})+c^{2} / 4}+d^{\prime} \leq \phi(\underline{y}+\underline{u}) \leq \phi(\underline{y})+2 a \sqrt{\phi(\underline{y})+c^{2} / 4}+d^{\prime \prime}
$$

valid for all $\underline{y} \in \Lambda_{M}$ and $\underline{u} \in[-1 / 2,1 / 2]^{k}$. It is then obvious that putting:

$$
M^{\prime}:=M-2 a \sqrt{M+c^{2} / 4}+d^{\prime} \text { and } M^{\prime \prime}:=M+2 a \sqrt{M+c^{2} / 4}+d^{\prime \prime}
$$

one has indeed:

$$
E_{M^{\prime}} \subset E_{M}^{\prime} \subset E_{M^{\prime \prime}} \Longrightarrow \mu\left(E_{M^{\prime}}\right) \leq \operatorname{card} \Lambda_{M} \leq \mu\left(E_{M^{\prime \prime}}\right),
$$


said otherwise:

$\frac{V_{k}}{D_{F}^{1 / 2}}\left(M-2 a \sqrt{M+c^{2} / 4}+d^{\prime}+\underline{y}_{0}^{2}\right)^{k / 2} \leq \operatorname{card} \Lambda_{M} \leq \frac{V_{k}}{D_{F}^{1 / 2}}\left(M+2 a \sqrt{M+c^{2} / 4}+d^{\prime \prime}+\underline{y}_{0}^{2}\right)^{k / 2}$.

Since, for all $\alpha, \beta, \gamma \in \mathbf{R}$, one has:

$$
(M+\alpha \sqrt{M+\beta}+\gamma)^{\frac{k}{2}}=M^{\frac{k}{2}}\left(1+O\left(M^{\frac{-1}{2}}\right)\right)^{\frac{k}{2}}=M^{\frac{k}{2}}+O\left(M^{\frac{k-1}{2}}\right),
$$

the desired estimation follows.

\section{Second auxiliary proposition.}

Proposition 5. For any $\alpha>0$ and $x \in \mathbf{C},|x|<1$, let $S_{\alpha}(x):=\sum_{n \geq 0} n^{\alpha} x^{n}$. Then, when $x \rightarrow 1^{-}:$

$$
S_{\alpha}(x)=\frac{\Gamma(\alpha+1)}{(1-x)^{\alpha+1}}+O\left(\frac{1}{(1-x)^{\alpha}}\right) .
$$

Proof. Write, for $0<x<1, a:=-\ln x>0$ and, for $t \geq 0, h_{a}(t):=t^{\alpha} x^{t}=t^{\alpha} e^{-a t}$. We thus have:

$$
I:=\int_{0}^{+\infty} h_{a}(t) d t=\frac{\Gamma(\alpha+1)}{a^{\alpha+1}}
$$

The function $h_{a}$ grows over $\left[0, \frac{\alpha}{a}\right]$ and decreases (with limit $0^{+}$) over $\left[\frac{\alpha}{a},+\infty[\right.$; its maximum is $h_{a}\left(\frac{\alpha}{a}\right)=\left(\frac{\alpha}{a e}\right)^{\alpha}$.

If $\alpha=a / m, m \in \mathbf{N}^{*}$, whence $\frac{\alpha}{a}=m$, one deduces the bounds:

$$
\begin{aligned}
h_{a}(0)+\cdots+h_{a}(m-1) & \leq \int_{0}^{m} h_{a}(t) d t \leq h_{a}(1)+\cdots+h_{a}(m) \\
h_{a}(m+1)+\cdots+h_{a}(n)+\cdots & \leq \int_{m}^{+\infty} h_{a}(t) d t \leq h_{a}(m)+\cdots+h_{a}(n)+\cdots,
\end{aligned}
$$

whence $\sum_{n \geq 0} h_{a}(n) \in\left[I-h_{a}(m), I+h_{a}(m)\right]$. Therefore:

$$
S_{\alpha}(x)=\frac{\Gamma(\alpha+1)}{(-\ln x)^{\alpha+1}}+O\left(\frac{1}{(-\ln x)^{\alpha}}\right)=\frac{\Gamma(\alpha+1)}{(1-x)^{\alpha+1}}+O\left(\frac{1}{(1-x)^{\alpha}}\right)
$$

for $\ln x \sim x-1$ when $x \rightarrow 1$. The estimation is therefore right for all $a:=\alpha / m$, but since $S_{\alpha}(x)$ is monotonous as a function of $a$, it stays valid without restriction when $a \rightarrow 0^{+}$ and $x \rightarrow 1^{-}$. 
Application to a generating series. $\quad$ Let $F$ and $L$ as defined at the beginning of 3.1 .2 and define the generating series:

$$
\Phi_{F, L}(x):=\sum_{\underline{m} \in \mathbf{Z}^{k}} x^{F(\underline{m})+L(\underline{m})}=\sum_{n \in Z} r_{F, L}(n) x^{n},
$$

where $r_{F, L}(n):=\operatorname{card}\left\{\underline{m} \in \mathbf{Z}^{k} \mid F(\underline{m})+L(\underline{m})=n\right\}$. The series $\Phi_{F, L}(x)$ only has a finite number of terms with negative exponent and it converges normally over every compact subset of the punctured open disk $\stackrel{\circ}{\mathbf{D}}(0,1) \backslash\{0\}$.

Proposition 6. For $x \rightarrow 1^{-}$, one has the asymptotic estimation:

$$
\Phi_{F, L}(x)=\frac{1}{D_{F}^{1 / 2}} \frac{\pi^{\frac{k}{2}}}{(1-x)^{\frac{k}{2}}}+O\left(\frac{1}{(1-x)^{\frac{k-1}{2}}}\right) .
$$

Proof. We abreviate $C:=\frac{V_{k}}{D_{F}^{1 / 2}} \cdot$ Then:

$\frac{\Phi_{F, L}(x)}{1-x}=\left(\sum_{n \in \mathbf{Z}} r_{F, L}(n) x^{n}\right)\left(\sum_{n \in \mathbf{Z}} x^{n}\right)=\sum_{n \in \mathbf{Z}} R_{F, L}(n) x^{n}$, or $R_{F, L}(n):=\sum_{p \leq n} r_{F, L}(p)=\operatorname{card} \Lambda_{n}$ with the same notations as before. From proposition 4, there is a constant $C^{\prime}>0$ such that:

$$
\forall n>0,\left|R_{F, L}(n)-C n^{\frac{k}{2}}\right|<C^{\prime} n^{\frac{k-1}{2}} .
$$

Let $\Psi(x):=\sum_{n>0} R_{F, L}(n) x^{n}$, so that, when $x \rightarrow 1^{-}$:

$$
\frac{\Phi_{F, L}(x)}{1-x}=\Psi(x)+O(1) .
$$

We immediately get, for $0<x<1$ :

$$
\left|\Psi(x)-C S_{\frac{k}{2}}(x)\right| \leq C^{\prime} S_{\frac{k-1}{2}}(x) .
$$

But, after proposition 5

$$
S_{\frac{k}{2}}(x)=\frac{\Gamma\left(\frac{k}{2}+1\right)}{(1-x)^{\frac{k}{2}+1}}+O\left(\frac{1}{(1-x)^{\frac{k}{2}}}\right) \text { and } S_{\frac{k-1}{2}}(x)=\frac{\Gamma\left(\frac{k-1}{2}+1\right)}{(1-x)^{\frac{k-1}{2}+1}}+O\left(\frac{1}{(1-x)^{\frac{k-1}{2}}}\right),
$$

whence:

$$
\frac{\Phi_{F, L}(x)}{1-x}=C \frac{\Gamma\left(\frac{k}{2}+1\right)}{(1-x)^{\frac{k}{2}+1}}+O\left(\frac{1}{(1-x)^{\frac{k+1}{2}}}\right) .
$$

Since we know that $V_{k}=\frac{\pi^{\frac{k}{2}}}{\Gamma\left(\frac{k}{2}+1\right)}$, the desired estimation follows. 


\subsubsection{End of the proof of theorem 1}

Odd and even parts of $f_{k, n}$. By partitioning $\mathbf{Z}^{k}$ into $2^{k}$ classes modulo the subgroup $(2 \mathbf{Z})^{k}$, one decomposes the generating series $f_{k, n}$ :

$$
\begin{aligned}
f_{k, n}(x) & =\sum_{\underline{m} \in \mathbf{Z}^{k}} x^{Q(\underline{m})-n S(\underline{m})} \\
& =\sum_{\underline{\varepsilon} \in\{0,1\}^{k}} \sum_{\underline{m} \in \mathbf{Z}^{k}} x^{Q(2 \underline{m}+\underline{\varepsilon})-n S(2 \underline{m}+\underline{\varepsilon})} \\
& =\sum_{\underline{\varepsilon} \in\{0,1\}^{k}} x^{Q(\underline{\varepsilon})-n S(\underline{\varepsilon})} \sum_{\underline{m} \in \mathbf{Z}^{k}} x^{4 Q(\underline{m})+4 B(\underline{m}, \underline{\varepsilon})-2 n S(\underline{m})},
\end{aligned}
$$

where $B$ denotes the symmetric bilinear form such that $B(\underline{m}, \underline{m})=Q(\underline{m})$. Each sum $\sum_{\underline{m} \in \mathbf{Z}^{k}} x^{4 Q(\underline{m})+4 B(\underline{m}, \underline{\varepsilon})-2 n S(\underline{m})}$ is even as a function of $\underline{m}$, because of the form of the exponents:

$$
4 Q(\underline{m})+4 B(\underline{m}, \underline{\varepsilon})-2 n S(\underline{m})=4 \sum_{1 \leq i \leq j \leq k} m_{i} m_{j}+2 \sum_{1 \leq i \leq j \leq k}\left(m_{i} \varepsilon_{j}+m_{j} \varepsilon_{i}\right)-2 n \sum_{1 \leq i \leq k} m_{i} .
$$

Therefore:

$$
\begin{aligned}
f_{k, n}(-x) & =\sum_{\underline{\varepsilon} \in\{0,1\}^{k}}(-1)^{Q(\underline{\varepsilon})-n S(\underline{\varepsilon})} x^{Q(\underline{\varepsilon})-n S(\underline{\varepsilon})} \sum_{\underline{m} \in \mathbf{Z}^{k}} x^{4 Q(\underline{m})+4 B(\underline{m}, \underline{\varepsilon})-2 n S(\underline{m})} \\
& =\sum_{\underline{\varepsilon} \in\{0,1\}^{k}}(-1)^{Q(\underline{\varepsilon})-n S(\underline{\varepsilon})} x^{Q(\underline{\varepsilon})-n S(\underline{\varepsilon})} \Phi_{Q, L_{\underline{\varepsilon}}}\left(x^{4}\right)
\end{aligned}
$$

where we use again the generating series $\Phi_{F, L}$ of (39), here with the quadratic form $F:=Q$ and the linear form $L:=L_{\underline{\varepsilon}}$ defined by:

$$
L_{\underline{\varepsilon}}(\underline{m}):=B(\underline{m}, \underline{\varepsilon})-(n / 2) S(\underline{m}) .
$$

Lemma 2. When $x \rightarrow 1^{-}$, we have:

$$
f(-x)=\left(\sum_{\underline{\varepsilon} \in\{0,1\}^{k}}(-1)^{Q(\underline{\varepsilon})-n S(\underline{\varepsilon})}\right) \frac{1}{2^{k} D_{Q}^{1 / 2}} \frac{\pi^{\frac{k}{2}}}{(1-x)^{\frac{k}{2}}}+O\left(\frac{1}{(1-x)^{\frac{k-1}{2}}}\right) .
$$

Proof. One calls upon proposition 6 and the fact that $\frac{1}{\left(1-x^{4}\right)^{\frac{k}{2}}} \sim \frac{1}{2^{k}(1-x)^{\frac{k}{2}}}$ when $x \rightarrow$ 1.

We are left with the task of determining the value (and the sign !) of the factor $\sum_{\underline{\varepsilon} \in\{0,1\}^{k}}(-1)^{Q(\underline{\varepsilon})-n S(\underline{\varepsilon})}$. 
Calculation of the factor $\sum_{\underline{\varepsilon} \in\{0,1\}^{k}}(-1)^{Q(\underline{\varepsilon})-n S(\underline{\varepsilon})}$ and conclusion. Let

$A:=\operatorname{card}\left\{\underline{\varepsilon} \in\{0,1\}^{k} \mid Q(\underline{\varepsilon}) \equiv n S(\underline{\varepsilon}) \quad(\bmod 2)\right\}$ and $B:=\operatorname{card}\left\{\underline{\varepsilon} \in\{0,1\}^{k} \mid Q(\underline{\varepsilon}) \not \equiv n S(\underline{\varepsilon}) \quad(\bmod 2)\right\}$.

One thus has:

$$
A-B=\sum_{\underline{\varepsilon} \in\{0,1\}^{k}}(-1)^{Q(\underline{\varepsilon})-n S(\underline{\varepsilon})} \text { and } A+B=2^{k} .
$$

If $l$ among the $k$ components $\varepsilon_{j}$ of $\underline{\varepsilon}$ have value 1 (so that $k-l$ have value 0 ), then $Q(\underline{\varepsilon})=l(l+1) / 2$ and $S(\underline{\varepsilon})=l$, whence:

$$
A=\sum_{\substack{0 \leq l \leq k \\
l(l+1) \\
\frac{2}{-n l \text { even }}}}\left(\begin{array}{l}
k \\
l
\end{array}\right) \text { and } B=\sum_{\substack{0 \leq l \leq k \\
\frac{l(l+1)}{2}-n l \text { odd }}}\left(\begin{array}{l}
k \\
l
\end{array}\right)
$$

We thus must discuss the parity of $l(l+1) / 2-n l$. If $n$ is even, it is the same as that of $l(l+1) / 2$, which is even if, and only if, the remainder of $l$ modulo 4 is 0 or 3 ; if $n$ is odd, it is the same as that of $l(l-1) / 2$, which is even if, and only if the remainder of $l$ modulo 4 is 0 or 1 . We are led to introduce:

$$
\forall j=0,1,2,3, s_{j}:=\sum_{\substack{0 \leq l \leq k \\
l \equiv j(\bmod 4)}}\left(\begin{array}{l}
k \\
l
\end{array}\right) .
$$

We then have:

$$
(A, B)=\left\{\begin{array}{l}
\left(s_{0}+s_{3}, s_{1}+s_{2}\right) \text { if } n \text { is even } \\
\left(s_{0}+s_{1}, s_{2}+s_{3}\right) \text { if } n \text { is odd }
\end{array}\right.
$$

The integers $s_{j}$ can be deduced from formulas (given here for $k>0$ ):

$$
\begin{aligned}
& s_{0}+s_{1}+s_{2}+s_{3}=(1+1)^{k}=2^{k}, \\
& s_{0}+\mathrm{i} s_{1}-s_{2}-\mathrm{i} s_{3}=(1+\mathrm{i})^{k}=2^{k / 2}(\cos k \pi / 4+\mathrm{i} \sin k \pi / 4) \text {, } \\
& s_{0}-s_{1}+s_{2}-s_{3}=(1-1)^{k}=0, \\
& s_{0}-\mathrm{i} s_{1}-s_{2}+\mathrm{i} s_{3}=(1-\mathrm{i})^{k}=2^{k / 2}(\cos k \pi / 4-\mathrm{i} \sin k \pi / 4) \text {. }
\end{aligned}
$$

One draws:

$$
\begin{aligned}
& s_{0}=\frac{1}{4}\left(2^{k}+2^{\frac{k}{2}+1} \cos k \pi / 4\right), \\
& s_{1}=\frac{1}{4}\left(2^{k}+2^{\frac{k}{2}+1} \sin k \pi / 4\right), \\
& s_{2}=\frac{1}{4}\left(2^{k}-2^{\frac{k}{2}+1} \cos k \pi / 4\right), \\
& s_{3}=\frac{1}{4}\left(2^{k}-2^{\frac{k}{2}+1} \sin k \pi / 4\right),
\end{aligned}
$$


whence, if $n$ is even:

$$
A-B=s_{0}-s_{1}-s_{2}+s_{3}=\frac{1}{4} 2^{\frac{k}{2}+1}(2 \cos k \pi / 4-2 \sin k \pi / 4)=2^{\frac{k+1}{2}} \cos (k+1) \pi / 4,
$$

and, if $n$ is odd:

$$
A-B=s_{0}+s_{1}-s_{2}-s_{3}=\frac{1}{4} 2^{\frac{k}{2}+1}(2 \cos k \pi / 4+2 \sin k \pi / 4)=2^{\frac{k+1}{2}} \cos (k-1) \pi / 4 .
$$

Combining these values with lemma 2, one finds:

$$
f_{k, n}(-x)=\frac{\pi^{\frac{k}{2}}}{2^{\frac{k-1}{2}} D_{Q}^{\frac{1}{2}}} \frac{\cos ((k+\varepsilon) \pi / 4)}{(1-x)^{\frac{k}{2}}}+O\left(\frac{1}{(1-x)^{\frac{k-1}{2}}}\right) .
$$

This formula can be made more precise by noting that $D_{Q}=(k+1) 2^{-k}$. (Quick argument: $a \in \mathbf{C}$ being fixed, let $D_{n}(x)$ the determinant of the $n \times n$ matrix having $x$ on the diagonal and $a$ elsewhere. One checks that $D_{n}^{\prime}(x)=n D_{n-1}(x)$ and $D_{n}(a)=0$, from which one deduces by induction that $D_{n}(x)=(x-a)^{n-1}(x+(n-1) a)$. One then sets $x:=1$ and $a:=1 / 2$.) Replacing $D_{Q}$ by $(k+1) 2^{-k}$ in the above formula:

$$
f_{k, n}(-x)=\frac{\pi^{\frac{k}{2}} \sqrt{2}}{\sqrt{k+1}} \frac{\cos ((k+\varepsilon) \pi / 4)}{(1-x)^{\frac{k}{2}}}+O\left(\frac{1}{(1-x)^{\frac{k-1}{2}}}\right) .
$$

This is indeed formula (36) of theorem 1 .

\subsubsection{Complement: a first attempt at exploiting modularity}

The generating series of the numbers of representations by positive definite quadratic form are known to have modular properties, it has been, since Jacobi, one of the clues of the appearance of theta functions in number theory. We shall here try (and only partially succeed) to exploit this fact to improve the above result.

Use of Poisson's summation formula. We use Poisson's formula in the form quoted in [4, §VII.6]. Let $f$ a function over $\mathbf{R}^{k}$, of Schwartz class, meaning that $f$ is $C^{\infty}$ and all its partial derivatives $D f$ at all orders have fast decay, i.e. they are such that $\forall N,|D f(\underline{x})|=$ $O\left(|\underline{x}|^{-N}\right)$ when $|\underline{x}| \rightarrow+\infty$. We write $\mu$ the Lebesgue measure and $\underline{x} \cdot \underline{y}$ the canonical inner product on $\mathbf{R}^{k}$. The Fourier transform $\hat{f}$, defined as:

$$
\hat{f}(\underline{y}):=\int_{\mathbf{R}^{k}} e^{-2 \mathrm{i} \pi \underline{x} \cdot \underline{y}} f(\underline{x}) d \mu(\underline{x})
$$

also is of Schwartz class; and Poisson's summation formula can be stated as follows:

$$
\sum_{\underline{m} \in \mathbf{Z}^{k}} \hat{f}(\underline{m})=\sum_{\underline{m} \in \mathbf{Z}^{k}} f(\underline{m})
$$


Now let $F(\underline{x}):=\sum a_{i, j} x_{i} x_{j}$ a positive definite quadratic form and $L(\underline{x}):=\sum b_{i} x_{i}$ a linear form over $\mathbf{R}^{k}$. The function:

$$
\phi_{F, L}(\underline{x}):=e^{-(F(\underline{x})+L(\underline{x}))}
$$

is of Schwartz class. We shall compute its Fourier transform. We write $X, Y, U, V, B, \ldots$ the column vectors associated with $\underline{x}, \underline{y}, \underline{u}, \underline{v}, \underline{b} \ldots$ and $A:=\left(a_{i, j}\right)$ the (real symmetric) matrix of $F$, so that $F(\underline{x})={ }^{t} X A X$. Likewise $L(\underline{x})={ }^{t} B X$, where $B$ is the column vector associated with $\underline{b}:=\left(b_{1}, \ldots, b_{k}\right)$. Let $S$ a real square invertible matrix such that $A={ }^{t} S S$ (such $S$ is known to exist) and let $T:=S^{-1}$. Then:

$$
\begin{aligned}
\widehat{\phi_{F, L}}(\underline{y}) & =\int_{\mathbf{R}^{k}} e^{-2 \mathrm{i} \pi \underline{x} \cdot \underline{y}-(F(\underline{x})+L(\underline{x}))} d \mu(\underline{x})=\int_{\mathbf{R}^{k}} e^{-{ }^{t} X A X-{ }^{t} B X-2 \mathrm{i} \pi^{t} Y X} d \mu(\underline{x}) \\
& =\frac{1}{|\operatorname{det} S|} \int_{\mathbf{R}^{k}} e^{-{ }^{t} U U-{ }^{t} B T U-2 \mathrm{i} \pi^{t} Y T U} d \mu(\underline{u}) \quad \text { by change of variable } X=S U \\
& =\frac{1}{|\operatorname{det} S|} \int_{\mathbf{R}^{k}} e^{-\underline{u}^{2}-\underline{v u}} d \mu(\underline{u}) \quad \text { where one put }{ }^{t} V:={ }^{t} B T+2 \mathrm{i} \pi^{t} Y T \\
& =\frac{e^{\underline{v}^{2}} / 4}{|\operatorname{det} S|} \int_{\mathbf{R}^{k}} e^{-(\underline{u}+\underline{v} / 2)^{2}} d \mu(\underline{u})=\frac{e^{\underline{v}^{2} / 4}}{|\operatorname{det} S|} \int_{\mathbf{R}^{k}} e^{-\underline{u}^{2}} d \mu(\underline{u})=\frac{\pi^{k / 2} e^{\underline{v}^{2} / 4}}{|\operatorname{det} S|} .
\end{aligned}
$$

(Because $\int_{\mathbf{R}^{k}} e^{-\underline{u}^{2}} d \mu(\underline{u})$ is the gaussian integral over $\mathbf{R}^{k}$.) At last, we compute:

$$
\underline{v}^{2}=\left({ }^{t} B T+2 \mathrm{i} \pi^{t} Y T\right)\left({ }^{t} T B+2 \mathrm{i} \pi^{t} T Y\right)=\left({ }^{t} B+2 \mathrm{i} \pi^{t} Y\right) A^{-1}(B+2 \mathrm{i} \pi Y)=\tilde{F}(\underline{b}+2 \mathrm{i} \pi \underline{y}),
$$

where was introduced the quadratic form $\tilde{F}$ with matrix $A^{-1}$ (this form also is positive definite). One has:

$$
\tilde{F}(\underline{b}+2 \mathrm{i} \pi \underline{y})=\tilde{F}(\underline{b})-4 \pi^{2}(\tilde{F}(\underline{y})+\tilde{L}(\underline{y})), \text { where } \tilde{L}(\underline{y}):=\frac{1}{\mathrm{i} \pi}^{t} B A^{-1} Y .
$$

And we eventually reaches the result:

$$
\widehat{\phi_{F, L}}(\underline{y})=\frac{\pi^{k / 2} e^{\tilde{F}(\underline{b} / 2)}}{\sqrt{\operatorname{det} A}} e^{-\pi^{2}(\tilde{F}(\underline{y})+\tilde{L}(\underline{y}))}
$$

Poisson's summation formula then yields:

$$
\sum_{\underline{m} \in \mathbf{Z}^{k}} e^{-(F(\underline{m})+L(\underline{m}))}=\frac{\pi^{k / 2} e^{\tilde{F}(\underline{b} / 2)}}{\sqrt{\operatorname{det} A}} \sum_{\underline{m} \in \mathbf{Z}^{k}} e^{-\pi^{2}(\tilde{F}(\underline{m})+\tilde{L}(\underline{m}))} .
$$

Application to a generating series. Recall that $f_{k, n}(x)=\sum_{\underline{m} \in \mathbf{Z}^{k}} x^{Q(\underline{m})-n S(\underline{m})}$ and that we are looking for conditions ensuring the vanishing of this function. We have:

$$
f_{k, n}(x)=\sum_{\underline{m} \in \mathbf{Z}^{k}} \phi_{F, L}(\underline{m}), \text { where } F:=t Q, L:=-t n S, \text { with } x=e^{-t} .
$$


Note that $|x|<1 \Leftrightarrow \mathfrak{R}(t)>0$. In order to apply formula 42, we thus take:

$$
A:=t\left(\begin{array}{cccc}
1 & 1 / 2 & \ldots & 1 / 2 \\
1 / 2 & 1 & \ldots & 1 / 2 \\
\vdots & \vdots & \ddots & \vdots \\
1 / 2 & 1 / 2 & \ldots & 1
\end{array}\right) \text { and } B:=-t n\left(\begin{array}{c}
1 \\
1 \\
\vdots \\
1
\end{array}\right)
$$

One easily checks that:

$$
A^{-1}=\frac{2}{(k+1) t}\left(\begin{array}{cccc}
k & -1 / 2 & \ldots & -1 \\
-1 & k & \ldots & -1 \\
\vdots & \vdots & \ddots & \vdots \\
-1 & -1 & \ldots & k
\end{array}\right)
$$

whence:

$\tilde{F}(\underline{y})=\frac{2}{(k+1) t} \tilde{Q}(\underline{y})$, where $\tilde{Q}(\underline{y}):=k \sum_{1 \leq i \leq k} y_{i}^{2}-2 \sum_{1 \leq i<j \leq k} y_{i} y_{j}, \quad$ and $\tilde{L}(\underline{y})=\frac{-2 n}{(k+1) \mathrm{i} \pi} S(\underline{y})$.

Setting $y:=e^{-\frac{2 \pi^{2}}{(k+1) t}}$, we then see that $f_{k, n}(x)$ equals, up to a non zero constant factor:

$$
g(y):=\sum_{\underline{m} \in \mathbf{Z}^{k}} e^{r i \pi S(\underline{m})} y^{\tilde{Q}(\underline{m})},
$$

where one put $r:=-\frac{2 n}{k+1} \in \mathbf{Q}$. The function $g(y)$ is well defined for $|y|<1$, which is consistent with the above definition of $y$ and the fact that $|x|<1 \Leftrightarrow \mathfrak{R}(t)>0 \Leftrightarrow \mathfrak{R}(1 / t)>$ 0 . Note that, since $\tilde{Q}$ is positive definite, $g(0)=1$. But the presence of a "phase" $\operatorname{ri} S(\underline{m})$ on the one hand, the arithmetical properties of $\tilde{Q}$ on the other hand, complicate slightly the use of real analysis here.

We follow, as far as possible, the same method as before:

$$
\begin{aligned}
g(y) & =\sum_{\underline{\varepsilon} \in\{0,1\}^{k}} \sum_{\underline{m} \in \mathbf{Z}^{k}} e^{r i \pi S(2 \underline{m}+\underline{\varepsilon})} y^{\tilde{Q}(2 \underline{m}+\underline{\varepsilon})} \\
& =\sum_{\underline{\varepsilon} \in\{0,1\}^{k}} e^{r i \pi S(\underline{\varepsilon})} y^{\tilde{Q}(\underline{\varepsilon})} \sum_{\underline{m} \in \mathbf{Z}^{k}} e^{2 r i \pi S(\underline{m})}\left(y^{4}\right)^{\tilde{Q}(\underline{m})+\tilde{B}(\underline{m}, \underline{\varepsilon})},
\end{aligned}
$$

where $\tilde{B}(\underline{u}, \underline{v}):=k \sum u_{i} v_{i}-2 \sum_{i<j}\left(u_{i} v_{j}+u_{j} v_{i}\right)$ denotes the symmetric bilinear form such that $\tilde{B}(\underline{m}, \underline{m})=\tilde{Q}(\underline{m})$. One also finds out that, if $\underline{\varepsilon}$ has $l$ coefficients with value 1 , then $S(\underline{\varepsilon})=l$ and $\tilde{Q}(\underline{\varepsilon})=k l-l(l-1)$. 
If $r$ is an integer. In that case, $e^{2 r i \pi S(\underline{m})}=1$, which somehow gets rid of phase problems. One want to make $y^{4} \rightarrow 1^{-}$but in such a way that $g(y)$ stays real. We will take $y=\mathrm{i}^{p} z$, $0 \leq z<1, p \in\{0,1,2,3\}$ being chosen such that $p k$ be even: $p k=2 s$. Then:

$$
y^{\tilde{Q}(\underline{\varepsilon})}=\mathrm{i}^{p k l-p l(l-1)} z^{\tilde{Q}(\underline{\varepsilon})}=(-1)^{s l-p l(l-1) / 2} z^{\tilde{Q}(\underline{\varepsilon})},
$$

and all terms are indeed real. Moreover, we have the following asymptotic estimate when $z \rightarrow 1^{-}$:

$$
g(y)=\left(\sum_{l=0}^{k}\left(\begin{array}{l}
k \\
l
\end{array}\right)(-1)^{r l+s l-p l(l-1) / 2}\right) \frac{C}{(1-z)^{k / 2}}+O\left(\frac{1}{(1-z)^{(k-1) / 2}}\right),
$$

where $C>0$ is computed as before using the discriminant of $\tilde{Q}$.

If the left factor of the main term is strictly negative, the limit of $g(y)$ is $-\infty$ and one deduces again the existence of a zero of $g(y)$. To compute said factor, one notes that $l(l-1) / 2$ is even if, and only if $l \equiv 0$ or $l \equiv 1(\bmod 4)$. One then has, with the same notations as before:

$$
\begin{aligned}
\sum_{l=0}^{k}\left(\begin{array}{l}
k \\
l
\end{array}\right)(-1)^{r l+s l-p l(l-1) / 2} & =s_{0}+(-1)^{s+r} s_{1}+(-1)^{p}\left(s_{2}+(-1)^{s+r} s_{3}\right) \\
& =\left(s_{0}+(-1)^{p} s_{2}\right)+(-1)^{s+r}\left(s_{1}+(-1)^{p} s_{3}\right) .
\end{aligned}
$$

If $p$ is even, since $s_{0}+s_{2}=s_{1}+s_{3}=2^{k-1}$, one finds 0 or $2^{k}$ and therefore certainly not a strictly negative number. So we suppose that $p$ is odd, i.e. $p=1$ or $p=3$. Either case amounts to the same, so we take $p=1$ and therefore $k=p k=2 s$. Since $r=-2 n /(k+1)$ has been assumed integral and since the denominator is odd, we see that $n$ is a multiple of $k+1$ and that $r$ is even.

Let us resume the calculation:

$$
\begin{aligned}
\sum_{l=0}^{k}\left(\begin{array}{l}
k \\
l
\end{array}\right)(-1)^{r l+s l-p l(l-1) / 2} & =\left(s_{0}-s_{2}\right)+(-1)^{s+r}\left(s_{1}-s_{3}\right) \\
& =2^{k / 2}\left(\cos k \pi / 4+(-1)^{s+r} \sin k \pi / 4\right) \\
& =2^{s}\left(\cos s \pi / 2+(-1)^{s+r} \sin s \pi / 2\right)
\end{aligned}
$$

This number is strictly negative if, and only if, $s \equiv 1$ or $2(\bmod 4)$. So we can conclude this study:

Proposition 7. If $k \equiv 2$ or $4(\bmod 8)$ and if $k+1 \mid n$, then $\gamma_{k, n}(q)$ vanishes for at least one value of $q$.

It is not easy here to characterize that value: one would require that the argument of $y:=e^{-\frac{2 \pi^{2}}{(k+1) t}}$ be $\pi / 2$, whence a condition on $t$ which we shall not try to write down. 
If $r^{\prime}:=r-1 / 2$ is an integer. In that case, one checks that $k$ is necessarily odd: $k=$ $2 s-1$. An analysis similar to the previous one leads us to take $y=\mathrm{i} z$, whence again $y^{4}=z^{4}$. With the same notations, one then gets:

$$
e^{r \mathrm{i} \pi S(\underline{\varepsilon})} y^{\tilde{Q}(\underline{\varepsilon})}=(-1)^{r^{\prime}+s l-l(l-1) / 2} z^{l(2 s-l)}
$$

and

$$
\sum_{\underline{m} \in \mathbf{Z}^{k}} e^{2 \operatorname{ri\pi } S(\underline{m})}\left(y^{4}\right)^{\tilde{Q}(\underline{m})+\tilde{B}(\underline{m}, \underline{\varepsilon})}=\sum_{\underline{m} \in \mathbf{Z}^{k}}(-1)^{S(\underline{m})}\left(z^{4}\right)^{\tilde{Q}(\underline{m})+\tilde{B}(\underline{m}, \underline{\varepsilon})} .
$$

It is the latter sum that causes problems: one can indeed check that the terms with $S(\underline{m})$ even, resp. odd, mutually compensate in such a way that the main term of the asymptotic estimation disappears. Our method does not allow to conclude in this case.

\subsection{Another approach to asymptotics when $q \rightarrow-1$}

Here again and until the end of the paper, we shall use the function $\vartheta$ and the coefficients $c_{k, n}(q)$ introduced in 2.2. We begin with a kind of "distribution formula".

Recall that $c_{1,0}(q)=1$. From now on, we shall assume that $k \geq 2$; also, from equation (15):

$$
\vartheta^{k}(x)=\sum_{n=0}^{k-1} c_{k, n}(q) x^{n} \vartheta_{q^{k}}\left(x^{k} q^{n}\right) .
$$

Last, recall the notation $\sum_{j(\bmod k)} f(j)$ which was introduced at the very end of section 1 .

Proposition 8. If $\mu$ is a primitive $k^{\text {th }}$ root of unity, one has:

$$
c_{k, n}(q)=\frac{q^{n^{2} / k} x^{-n}}{k \vartheta_{q^{k}}\left(x^{k}\right)} \sum_{j(\bmod k)} \mu^{-n j} \vartheta^{k}\left(x q^{-n / k} \mu^{j}\right)
$$

In particular:

$$
c_{k, n}(q)=\frac{q^{n^{2} / k}}{k \vartheta_{q^{k}}(1)} \sum_{j(\bmod k)} e^{2 n j \pi i / k} \vartheta^{k}\left(q^{n / k} e^{2 j \pi i / k}\right) .
$$

Proof. For $r \in\{0, \ldots, k-1\}$ and $j \in \mathbf{Z}$, divide by $x^{r}$ both members of (15) and then replace $x$ by $x \mu^{j}$; it follows that:

$$
x^{-r} \mu^{-r j} \vartheta^{k}\left(x \mu^{j}\right)=\sum_{n=0}^{k-1} c_{k, n}(q) x^{n-r} \mu^{(n-r) j} \vartheta_{q^{k}}\left(x^{k} q^{n}\right) .
$$

With $r=n$ and index $j$ running along a complete system of integers modulo $k$, we find that coefficients $c_{k, n}(q)$ satisfy the following identities:

$$
x^{-n} \sum_{j(\bmod k)} \mu^{-n j} \vartheta^{k}\left(x \mu^{j}\right)=k c_{k, n}(q) \vartheta_{q^{k}}\left(x^{k} q^{n}\right) .
$$


Substituting $x q^{-n / k}$ for $x$ above, we deduce (43).

Putting $x=1$ and $\mu=e^{-2 \pi i / k}$ in (43), we immediately obtain (44).

Note that, since $\vartheta\left(q^{-n / k} \mu^{j}\right)=\vartheta\left(q^{n / k} \mu^{-j}\right)=q^{1 / 2-n / k} \mu^{j} \vartheta\left(q^{(n-k) / k} \mu^{-j}\right)$, one can put (44) under the following form:

$$
c_{k, n}(q)=\frac{q^{\left((k-n)^{2}+n^{2}\right) /(2 k)}}{k \vartheta_{q^{k}}(1)} \sum_{j=0}^{k-1} \mu^{(k-n) j} \vartheta^{k}\left(q^{(n-k) / k} \mu^{-j}\right) .
$$

Comparing it with formula (44) where one has replaced $n$ by $k-n$, one recovers the symmetry property of $c_{k, n}$ already encountered.

\subsubsection{Expression as a finite sum of theta quotients}

We shall now consider, when $q \rightarrow-1$, the asymptotic behaviour of $c_{k, n}(q)$. We shall for that use the modular transformation of $\vartheta$. So we borrow from [2, p. 166, (76.1)] the notation $\vartheta_{3}(v \mid \tau)$ :

$$
\vartheta_{3}(v \mid \tau):=\sum_{n \in \mathbf{Z}} e^{\pi \mathrm{i}\left(n^{2} \tau+2 n v\right)}
$$

whence

$$
\vartheta_{q}(x)=\vartheta_{3}(v \mid \tau) \text { for } x=e^{2 \pi \mathrm{i} v} \text { and } q=e^{2 \pi \mathrm{i} \tau} .
$$

Let $M:=\left(\begin{array}{cc}-1 & 0 \\ 2 & -1\end{array}\right), \tau^{\prime}:=M \tau=\frac{-\tau}{2 \tau-1}$ and $q^{\prime}:=e^{2 \pi \mathrm{i} \tau^{\prime}}$. If $a=-1, b=0, c=2$ and $d=-1$, note that $\vartheta_{1-a-c, 1-b-d}=\vartheta_{0,2}=\vartheta_{3}$, where $\vartheta_{\mu, v}$ is defined in [2, p. 181, (81.2)]. From that, the modular formula given for $\vartheta_{3}$ in [2, p. 182, Theorem] implies that:

$$
\vartheta_{q}(x)=\frac{\mathrm{i}}{\varepsilon_{2}} e^{-2 \pi \mathrm{i} v v^{\prime}} \sqrt{\frac{\mathrm{i}}{2 \tau-1}} \vartheta_{q^{\prime}}\left(x^{\prime}\right)
$$

where $\varepsilon_{2}:=e^{\pi \mathrm{i} / 4}\left(\frac{2}{-1}\right), x:=e^{2 \pi \mathrm{i} v}, v^{\prime}=\frac{v}{2 \tau-1}$ and $x^{\prime}=e^{2 \pi \mathrm{i} v^{\prime}}$. Since $\left(\frac{2}{-1}\right)=\left(\frac{2}{1}\right)=$ 1 , one deduces that:

$$
\vartheta_{q}(x)=e^{2 \pi \mathrm{i}\left(1 / 8-v v^{\prime}\right)} \sqrt{\frac{\mathrm{i}}{2 \tau-1}} \vartheta_{q^{\prime}}\left(x^{\prime}\right)
$$

Recall that $\gamma_{k, n}(q)=c_{k, n}(q) q^{-n / 2}$.

Proposition 9. The following formula holds:

$$
\gamma_{k, n}(q)=\frac{e^{\pi i(k-2 n) / 4}\left(q e^{-\pi i}\right)^{n(n-k) /(2 k)}}{k \vartheta_{q^{k}}(1)}\left(\frac{i}{2 \tau-1}\right)^{k / 2} S_{k, n}(q),
$$

where

$$
S_{k, n}(q):=\sum_{j(\bmod k)}\left(q^{\prime} e^{\pi i}\right)^{(2 j+n)^{2} /(2 k)} \vartheta_{q^{\prime}}^{k}\left(q^{\prime(2 j+n) / k} e^{2 \pi i j / k}\right) .
$$


Proof. Note that $\tau=\frac{\tau^{\prime}}{2 \tau^{\prime}+1}$ and $(2 \tau-1)\left(2 \tau^{\prime}+1\right)=-1$. For $x:=q^{n / k} e^{2 j \pi \mathrm{i} / k}$, write $v:=\frac{n \tau+j}{k}$ so that:

$$
v=\frac{n}{2 k}(2 \tau-1)+\frac{2 j+n}{2 k}, \quad v^{\prime}=\frac{n}{2 k}+\frac{2 j+n}{2 k(2 \tau-1)}=-\frac{j}{k}-\frac{2 j+n}{k} \tau^{\prime}
$$

and

$$
v v^{\prime}=\frac{n^{2}}{4 k^{2}}(2 \tau-1)+\frac{n(2 j+n)}{2 k^{2}}+\frac{(2 j+n)^{2}}{4 k^{2}(2 \tau-1)}=\frac{n^{2} \tau}{2 k^{2}}-\frac{j^{2}}{k^{2}}-\frac{(2 j+n)^{2} \tau^{\prime}}{2 k^{2}} .
$$

Applying (45) to $x=q^{n / k} e^{2 j \pi i / k}$ and considering relation $\vartheta_{q^{\prime}}\left(x^{\prime}\right)=\vartheta_{q^{\prime}}\left(1 / x^{\prime}\right)$, one gets:

$$
\vartheta_{q}^{k}\left(q^{n / k} e^{2 j \pi \mathrm{i} / k}\right)=C_{j}\left(\frac{i}{2 \tau-1}\right)^{k / 2} q^{\prime(2 j+n)^{2} /(2 k)} \vartheta_{q^{\prime}}^{k}\left(q^{\prime(2 j+n) / k} e^{2 \pi i j / k}\right),
$$

where $C_{j}:=e^{2 \pi \mathrm{i}\left(k / 8-\left(n^{2} \tau-2 j^{2}\right) /(2 k)\right)}$. Since

$$
e^{2 n j \pi \mathrm{i} / k} C_{j}=e^{2 \pi \mathrm{i}\left(k / 8-\left(n^{2}-(2 j+n)^{2}\right) /(4 k)\right)} q^{-n^{2} /(2 k)},
$$

the relations (44) and (48) imply:

$$
\begin{aligned}
c_{k, n}(q)= & \frac{e^{\pi i k / 4}\left(q e^{-\pi \mathrm{i}}\right)^{n^{2} /(2 k)}}{k \vartheta_{q^{k}}(1)}\left(\frac{\mathrm{i}}{2 \tau-1}\right)^{k / 2} \times \\
& \sum_{j(\bmod k)}\left(q^{\prime} e^{\pi \mathrm{i}}\right)^{(2 j+n)^{2} /(2 k)} \vartheta_{q^{\prime}}^{k}\left(q^{\prime(2 j+n) / k} e^{2 \pi \mathrm{i} j / k}\right),
\end{aligned}
$$

which is plainly equivalent to (46).

\subsubsection{Sufficient conditions for vanishing at real negative values}

The main goal of all the rest of this section is the proof of the following result.

Theorem 2. Let $k$ an integer $\geq 3$ and $n$ an integer lying between 0 and $k-1$, and suppose that $q$ tends to -1 within the real interval $]-1,0[$.

- One has $\lim _{q \rightarrow-1} \gamma_{k, n}(q)=0$ if, and only if, $k$ is even and $k-2 n \equiv 2(\bmod 4)$.

- Otherwise, $\lim _{q \rightarrow-1}\left|\gamma_{k, n}(q)\right|=+\infty$. More precisely, one has $\lim _{q \rightarrow-1}(-1)^{k^{\prime}-n^{\prime}} \gamma_{k, n}(q)=$ $+\infty$ if $(k, n)$ can be expressed by means of a pair of integers $\left(k^{\prime}, n^{\prime}\right)$ in one of the following manners:

1. $k=4 k^{\prime}, n=2 n^{\prime}$; 
2. $k=4 k^{\prime}+2, n=2 n^{\prime}+1$;

3. $k=4 k^{\prime}+1, n \in\left\{2 n^{\prime}, 2 n^{\prime}+1\right\}$;

4. $k=4 k^{\prime}-1, n \in\left\{2 n^{\prime}, 2 n^{\prime}-1\right\}$.

Proof of theorem 2 comes through a series of lemmas and remarks. We now give a direct consequence on zeroes of $\gamma_{k, n}(q)$. Assume that $k \geq 3$ and $n$ lies between 0 and $k-1$. Applying theorem 2, we find that $\gamma_{k, n}(q) \rightarrow-\infty$ for $q \rightarrow-1$ only in the following cases:

(i) $k \equiv 0$ or $4(\bmod 8)$ and $n \equiv 2$ or $0(\bmod 4)$ respectively;

(ii) $k \equiv 2$ or $6(\bmod 8)$ and $n \equiv 3$ or $1(\bmod 4)$ respectively;

(iii) $k \equiv 1$ or $5(\bmod 8)$ and $n \equiv 2,3$ or $0,1(\bmod 4)$ respectively;

(iv) $k \equiv 3$ or $7(\bmod 8)$ and $n \equiv 0,3$ or $2,1(\bmod 4)$ respectively.

In all these cases $\gamma_{k, n}(q)$ admits a zero somewhere over $]-1,0[$. From that point on, we easily recover corollary 2 and its consequences.

\subsubsection{Application of modular forms to $\vartheta_{q^{k}}(1)$}

Suppose that $\tau \rightarrow \frac{1}{2}$ and $q \rightarrow-1$. More precisely, write $\tau=\frac{1}{2}+\mathrm{i} t$ with $t>0$; then:

$$
q=e^{\pi \mathrm{i}-2 \pi t}, \quad \tau^{\prime}=-\frac{1}{2}+\frac{\mathrm{i}}{4 t}, \quad q^{\prime}=e^{-\pi \mathrm{i}-\pi / 2 t} .
$$

It follows that $q^{\prime}$ will tend to 0 exponentally fast as $t \rightarrow 0^{+}$. For the sake of simplicity shall denote $\varnothing$ any function $f$ defined over $] 0,+\infty\left[\right.$ and such that $f(t)=O\left(e^{-\kappa / t}\right)$ for $t \rightarrow 0$ for some $\kappa>0$. This being said, taking $x:=1$ in (45), one gets:

$$
\vartheta_{q}(1)=\frac{e^{\pi \mathrm{i} / 4}}{\sqrt{2 t}}(1+\varnothing) .
$$

This will be generalized as follows.

Lemma 3. Let $k \in \mathbf{N}^{*}$. If $q=e^{\pi i-2 \pi t}$ with $t \rightarrow 0^{+}$, we have:

$$
\vartheta_{q^{k}}(1)=\left\{\begin{array}{lll}
\frac{1}{\sqrt{k t}}(1+\varnothing) & k \equiv 0 & (\bmod 4) ; \\
\frac{e^{\pi i / 4}}{\sqrt{2 k t}}(1+\varnothing) & k \equiv 1 & (\bmod 4) ; \\
\frac{2}{\sqrt{k t}} e^{-1 /(4 k t)}(1+\varnothing) & k \equiv 2 & (\bmod 4) ; \\
\frac{e^{-\pi i / 4}}{\sqrt{2 k t}}(1+\varnothing) & k \equiv 3 & (\bmod 4) .
\end{array}\right.
$$


Proof. We first write $\vartheta_{q^{k}}(1)=\vartheta_{3}(0 \mid k \tau)=\vartheta_{3}\left(0 \mid \frac{k}{2}+k t i\right)$. Taking in account formula [2, p.181, (81.12)], we obtain the following expressions:

$$
\vartheta_{q^{k}}(1)=\left\{\begin{array}{lll}
\vartheta_{3}(0 \mid k t \mathrm{i}) & k \equiv 0 & (\bmod 4) ; \\
\vartheta_{3}\left(0 \mid \frac{1}{2}+k t \mathrm{i}\right) & k \equiv 1 & (\bmod 4) \\
\vartheta_{4}(0 \mid k t \mathrm{i}) & k \equiv 2 & (\bmod 4) \\
\vartheta_{4}\left(0 \mid \frac{1}{2}+k t \mathrm{i}\right) & k \equiv 3 & (\bmod 4) .
\end{array}\right.
$$

If $k \equiv 0(\bmod 4)$, looking at the first relation of $(52)$ and the second modular relation from [2, p. 177, (79.9)] implies that

$$
\vartheta_{3}(0 \mid k t \mathrm{i})=\frac{1}{\sqrt{k t}} \vartheta_{3}\left(0 \mid \frac{\mathrm{i}}{k t}\right)=\frac{1}{\sqrt{k t}} \sum_{n \in \mathbf{Z}} e^{-\pi n^{2} /(k t)},
$$

whence the first asymptotic estimation stated in (51).

If $k \equiv 1(\bmod 4)$, one obtains the corresponding asymptotic estimate (51) from replacing $t$ by $k t$ in (50).

If $k \equiv 2(\bmod 4)$, consider the third relation in (52) and the first modular relation in [2, p. 177, (79.9)]: this implies that

$$
\vartheta_{4}(0 \mid k t \mathrm{i})=\frac{1}{\sqrt{k t}} \vartheta_{2}\left(0 \mid \frac{i}{k t}\right)=\frac{1}{\sqrt{k t}} \sum_{n \in \mathbf{Z}} e^{-\pi(n+1 / 2)^{2} /(k t)},
$$

where $\vartheta_{2}$ is defined in [2, p.166, (76.1)]. One deduces:

$$
\vartheta_{4}(0 \mid k t \mathrm{i})=\frac{1}{\sqrt{k t}}\left(2 e^{-\pi /(4 k t)}+2 e^{-9 \pi /(4 k t)}+\cdots\right)=\frac{2}{\sqrt{k t}} e^{-\pi /(4 k t)}(1+\varnothing),
$$

which yields the third formula in (51).

Last, if $k \equiv 3(\bmod 4)$, we shall use the last relation of (52). Applying the last modular formula from [2, p. 182 , Theorem] to the quadruple $(a, b, c, d)=(-1,0,2,-1)$, we see that, for $\tau^{\prime}=\frac{-\tau}{2 \tau-1}$ :

$$
\vartheta_{4}\left(v^{\prime} \mid \tau^{\prime}\right)=\mathrm{i}^{2} \varepsilon_{2} \sqrt{\frac{2 \tau-1}{\mathrm{i}}} e^{2 \pi \mathrm{i} v v^{\prime}} \vartheta_{2,1}(v \mid \tau)
$$

where, as before, $\varepsilon_{2}=e^{\pi \mathrm{i} / 4}$ and $v, v^{\prime}$ are the same as in (45). Modifying the first relation from [2, p. 181, (81.31)] into the form $\vartheta_{\mu+2, v}(v \mid \tau)=(-1)^{v} \vartheta_{\mu, v}(v \mid \tau)$, we find that $\vartheta_{2,1}(v \mid \tau)=-\vartheta_{0,1}(v \mid \tau)=-\vartheta_{4}(v \mid \tau)$. Putting $v=0$ and $\tau=\frac{1}{2}+t$ i hereabove, one draws that

$$
\vartheta_{4}\left(0 \mid \frac{1}{2}+t \mathrm{i}\right)=\frac{e^{-\pi \mathrm{i} / 4}}{\sqrt{2 t}} \vartheta_{4}\left(0 \mid-\frac{1}{2}+\frac{\mathrm{i}}{t}\right)
$$


Expanding $\vartheta_{4}(0 \mid \tau)$ with the help of [2, p.166, (76.1)], we have:

$$
\vartheta_{4}\left(0 \mid-\frac{1}{2}+\frac{\mathrm{i}}{t}\right)=\sum_{n \in \mathbf{Z}}(-1)^{n} e^{-\pi \mathrm{i} n^{2} / 2-\pi n^{2} / t}=1+\varnothing .
$$

Substituting $k t$ for $t$ in the last relation, we eventually obtain the complete asymptotic estimation (51).

Combining (51) with (46), we deduce the following remark.

Remark 3. Let $k$ and $n$ integers such that $0 \leq n<k$ and let $\Gamma_{k, n}$ the function defined over ] $0,+\infty[$ by the relation

$$
\Gamma_{k, n}(t):=k^{-1 / 2} 2^{-k / 2} e^{\pi n(n-k) t / k} t^{(1-k) / 2} .
$$

If $q=e^{\pi i-2 \pi t}$ with $t \rightarrow 0^{+}$, one has:

$$
\gamma_{k, n}(q)=\left\{\begin{array}{lll}
e^{\pi i(k-2 n) / 4} \Gamma_{k, n}(t) S_{k, n}(q)(1+\varnothing) & k \equiv 0 & (\bmod 4) ; \\
\sqrt{2} e^{\pi i(k-1-2 n) / 4} \Gamma_{k, n}(t) S_{k, n}(q)(1+\varnothing) & k \equiv 1 & (\bmod 4) ; \\
\frac{1}{2} e^{\pi i(k-2 n) / 4} \Gamma_{k, n}(t) S_{k, n}(q) e^{\pi /(4 k t)}(1+\varnothing) & k \equiv 2 & (\bmod 4) ; \\
\sqrt{2} e^{\pi i(k+1-2 n) / 4} \Gamma_{k, n}(t) S_{k, n}(q)(1+\varnothing) & k \equiv 3 & (\bmod 4) .
\end{array}\right.
$$

\subsubsection{Asymptotic study of theta functions with modular variables}

We now are left to study the asymptotic behaviour of $S_{k, n}(q)$ for $q \rightarrow-1$. In order to do so, let us consider expression (47), and put:

$$
s_{\alpha}(q)=s_{k, n, \alpha}(q)=\left(q^{\prime} e^{\pi \mathrm{i}}\right)^{\alpha^{2} / 2} \vartheta_{q^{\prime}}\left(\left(q^{\prime} e^{\pi \mathrm{i}}\right)^{\alpha} e^{-\pi \mathrm{i} n / k}\right)
$$

for every $\alpha \in \mathbf{R}$. Since $\vartheta_{q}(x)=0$ for $x \in-q^{\frac{1}{2}+\mathbf{Z}}$, we draw the following remark:

Remark 4. If $n / k=1 / 2$ and $\alpha=-1 / 2$, one has:

$$
s_{-1 / 2}(q)=0 .
$$

Expanding $\vartheta_{q^{\prime}}$ in series with $q^{\prime}=e^{-\pi i-\pi /(2 t)}$ (see (49)), we have:

$$
s_{\alpha}(q)=\sum_{m \in \mathbf{Z}} e^{-\pi i m(m / 2+n / k)-\pi(\alpha+m)^{2} /(4 t)},
$$

which implies that $s_{\alpha+2}(q)=s_{\alpha}(q)$. 
So let us make up a "fundamental" system with $k$ elements as follows:

$$
\Lambda_{k, n} \equiv\left\{\frac{2 j+n}{k} \quad(\bmod 2): j \in \mathbf{Z}\right\}
$$

More precisely, we will choose $\Lambda_{k, n}$ in the following way:

$$
\Lambda_{k, n}=\left\{\begin{array}{lll}
\left\{-1+\frac{2}{k}, \ldots, 0, \ldots, 1\right\} & (k, n) \equiv(0,0) & (\bmod 2) ; \\
\left\{-1+\frac{1}{k}, \ldots, \frac{1}{k}, \ldots, 1-\frac{1}{k}\right\} & (k, n) \equiv(0,1) & (\bmod 2) ; \\
\left\{-1+\frac{1}{k}, \ldots, 0, \ldots, 1-\frac{1}{k}\right\} & (k, n) \equiv(1,0) & (\bmod 2) ; \\
\left\{-1+\frac{2}{k}, \ldots, \frac{1}{k}, \ldots, 1\right\} & (k, n) \equiv(1,1) & (\bmod 2) .
\end{array}\right.
$$

When $\alpha$ runs in $\Lambda_{k, n}$, the terms $s_{\alpha}$ defined in (55) give rise to the following identity, coming from (47):

$$
S_{k, n}(q)=\sum_{\alpha \in \Lambda_{k, n}} s_{\alpha}^{k}(q)
$$

Successively taking $\alpha:=0$ and 1 in expression (57), one finds that:

$$
s_{0}(q)=1+2 \sum_{m=1}^{\infty} \cos (m n \pi / k) e^{-\pi \mathrm{i} m^{2} / 2-\pi m^{2} /(4 t)}
$$

and:

$$
s_{1}(q)=e^{\pi \mathrm{i}(n / k-1 / 2)}\left(1+2 \sum_{m=1}^{\infty}(-1)^{m} \cos (m n \pi / k) e^{-\pi \mathrm{i} m^{2} / 2-\pi m^{2} /(4 t)}\right) .
$$

One deduces that:

$$
s_{0}^{k}(q)=1-2 k \mathrm{i} \cos (n \pi / k) e^{-\pi /(4 t)}+O\left(e^{-\pi /(2 t)}\right)
$$

and

$$
s_{1}^{k}(q)=e^{\pi \mathrm{i}(n-k / 2)}\left(1+2 k \mathrm{i} \cos (n \pi / k) e^{-\pi /(4 t)}+O\left(e^{-\pi /(2 t)}\right)\right) .
$$

Let $\alpha \in] 0,1\left[\right.$ and put $\alpha^{\prime}:=\min \left((\alpha-2)^{2},(\alpha+1)^{2}\right)>1$; we shall assume that $k \geq 3$. Using expression (57), we obtain that

$$
s_{\alpha}(q)=e^{-\pi \alpha^{2} /(4 t)}-\mathrm{i} e^{\pi \mathrm{i} n / k-\pi(1-\alpha)^{2} /(4 t)}+O\left(e^{-\pi \alpha^{\prime} /(4 t)}\right)
$$


and

$$
s_{-\alpha}(q)=e^{-\pi \alpha^{2} /(4 t)}-\mathrm{i} e^{-\pi \mathrm{i} n / k-\pi(1-\alpha)^{2} /(4 t)}+O\left(e^{-\pi \alpha^{\prime} /(4 t)}\right) .
$$

In particular, if $\alpha=1 / k$, then $\alpha^{\prime}=(k+1)^{2} / k^{2}$; taking in account relations $(1-\alpha)^{2}-\alpha^{2}=$ $(k-2) / k$ and $\alpha^{\prime}-\alpha^{2}=(k+2) / k$ entails:

$$
s_{1 / k}(q)=e^{-\pi /\left(4 k^{2} t\right)}\left(1-\mathrm{i} e^{\pi \mathrm{i} n / k-\pi(k-2) /(4 k t)}+O\left(e^{-\pi(k+2) /(4 k t)}\right)\right)
$$

and

$$
s_{-1 / k}(q)=e^{-\pi /\left(4 k^{2} t\right)}\left(1-\mathrm{i} e^{-\pi \mathrm{i} n / k-\pi(k-2) /(4 k t)}+O\left(e^{-\pi(k+2) /(4 k t)}\right)\right) .
$$

If $k^{*}=\min (2 k-4, k+2)$, one gets that:

(62)

$$
s_{1 / k}^{k}(q)+s_{-1 / k}^{k}(q)=2 e^{-\pi /(4 k t)}\left(1-2 k \mathrm{i} \cos (n \pi / k) e^{-\pi(k-2) /(4 k t)}+O\left(e^{-\pi k^{*} /(4 k t)}\right)\right) .
$$

In a similar way, one will find that:

$$
\begin{aligned}
s_{1-1 / k}^{k}(q)+s_{-1+1 / k}^{k}(q)= & (-1)^{k+n} 2 i^{k} e^{-\pi /(4 k t)} \times \\
& \left(1+2 k i \cos (n \pi / k) e^{-\pi(k-2) /(4 k t)}+O\left(e^{-\pi k^{*} /(4 k t)}\right)\right) .
\end{aligned}
$$

Moreover, if $k \geq 4$ and $\alpha \in\left[\frac{2}{k}, 1-\frac{2}{k}\right]$ :

$$
s_{\alpha}^{k}(q)+s_{-\alpha}^{k}(q)=O\left(e^{-\pi /(k t)}\right) .
$$

Proposition 10. Let $k \geq 3$ and $0 \leq n<k$. The following asymptotic estimates hold $S_{k, n}(q)$ whenever $q=e^{\pi i-2 \pi t}$ and $t \rightarrow 0^{+}$:

1. If $(k, n) \equiv(0,0)(\bmod 2)$ :

$$
S_{k, n}(q)=2 e^{\pi i(2 n-k) / 4} \cos \frac{(2 n-k) \pi}{4}+O\left(e^{-\pi /(k t)}\right) .
$$

2. If $(k, n) \equiv(0,1)(\bmod 2)$ :

$$
S_{k, n}(q)=2\left(1-i^{k}\right) e^{-\pi /(4 k t)}+O\left(e^{-\pi \min (k-2,4) /(4 k t)}\right) .
$$

3. If $(k, n) \equiv(1,0)(\bmod 2)$ :

$$
S_{k, n}(q)=1-2 i^{k} e^{-\pi /(4 k t)}+O\left(e^{-\pi \min (k-1,4) /(4 k t)}\right) .
$$

4. If $(k, n) \equiv(1,1)(\bmod 2)$ :

$$
S_{k, n}(q)=i^{k}+2 e^{-\pi /(4 k t)}+O\left(e^{-\pi \min (k-1,4) /(4 k t)}\right) .
$$


Proof. We shall use the expression of $S_{k, n}(q)$ given in (59) with the various forms of $\Lambda_{k, n}$ shown in (58).

(1) Suppose that $(k, n) \equiv(0,0)(\bmod 2)$; one has $k \geq 4$. Because of the first relation of (58), one can write (59) in the following form:

$$
S_{k, n}(q)=s_{0}^{k}(q)+s_{1}^{k}(q)+\sum_{j=1}^{\frac{k}{2}-1}\left(s_{2 j / k}^{k}(q)+s_{-2 j / k}^{k}(q)\right)
$$

Consider (60), (61) together with (64), and note that $\frac{1}{2} \geq \frac{2}{k}$; then:

$$
S_{k, n}(q)=1+e^{\pi \mathrm{i}(n-k / 2)}+O\left(e^{-\pi /(k t)}\right),
$$

which takes us to relation (65).

(2) Suppose that $(k, n) \equiv(0,1)(\bmod 2)$; again, $k \geq 4$. Thanks to the second relation of (58), expression (59) can be put in the following form:

$$
\begin{aligned}
S_{k, n}(q)= & \left(s_{1 / k}^{k}(q)+s_{-1 / k}^{k}(q)\right)+\left(s_{1-1 / k}^{k}(q)+s_{1 / k-1}^{k}(q)\right) \\
& +\sum_{j=1}^{\frac{k}{2}-2}\left(s_{(2 j+1) / k}^{k}(q)+s_{-(2 j+1) / k}^{k}(q)\right) .
\end{aligned}
$$

Since $(-1)^{k+n} \mathrm{i}^{k}=-\mathrm{i}^{k}$, one obtains directly (66), taking in account relations (62), (63) together with (64).

(3) Suppose that $(k, n) \equiv(1,0)(\bmod 2)$. By virtue of the third relation of $(58)$, one transforms (59) into:

$$
S_{k, n}(q)=s_{0}^{k}(q)+\left(s_{1-1 / k}^{k}(q)+s_{1 / k-1}^{k}(q)\right)+\sum_{j=1}^{\frac{k-3}{2}}\left(s_{2 j / k}^{k}(q)+s_{-2 j / k}^{k}(q)\right) .
$$

Relation (67) flows immediately from the estimations contained in (60), (63) combined with (64).

(4) Last, suppose that $(k, n) \equiv(1,1)(\bmod 2)$. By virtue of the last relation of $(\underline{58})$, one expresses (59) in the following form:

$$
S_{k, n}(q)=s_{1}^{k}(q)+\left(s_{1 / k}^{k}(q)+s_{-1 / k}^{k}(q)\right)+\sum_{j=1}^{\frac{k-3}{2}}\left(s_{(2 j+1) / k}^{k}(q)+s_{-(2 j+1) / k}^{k}(q)\right) .
$$

Meanwhile, also note that $e^{\pi \mathrm{i}(n-k / 2)}=(-1)^{n-k} \mathrm{i}^{k}=\mathrm{i}^{k}$. By way of consequence, relation (68) directly follows from formulas (61), (62) with (64).

\subsubsection{End of the proof of theorem 2}

Considering remark 3 together with proposition 10 , we obtain the following result. 
Theorem 3. Let $k \geq 3$ and $0 \leq n<k$ and let $\Gamma_{k, n}$ the function defined in (53). We have the following asymptotic estimations for $\gamma_{k, n}(q)$ when $q=e^{\pi i-2 \pi t}$ and $t \rightarrow 0^{+}$:

1. If $k$ is even and $k-2 n \equiv 2(\bmod 4)$, then $\gamma_{k, n}(q)=\varnothing$.

2. If $k=4 k^{\prime}$ and $n=2 n^{\prime}$ or if $k=4 k^{\prime}+2$ and $n=2 n^{\prime}+1$, where $k^{\prime}, n^{\prime} \in \mathbf{Z}$, then:

$$
\gamma_{k, n}(q)=(-1)^{k^{\prime}-n^{\prime}} 2 \Gamma_{k, n}(t)(1+\varnothing) \text {. }
$$

3. If $k=4 k^{\prime}+1$ and $n \in\left\{2 n^{\prime}, 2 n^{\prime}+1\right\}$ or if $k=4 k^{\prime}-1$ and $n \in\left\{2 n^{\prime}, 2 n^{\prime}-1\right\}$, where $k^{\prime}, n^{\prime} \in \mathbf{Z}$, then:

$$
\gamma_{k, n}(q)=(-1)^{k^{\prime}-n^{\prime}} \sqrt{2} \Gamma_{k, n}(t)(1+\varnothing)
$$

Proof. Suppose first that $k \equiv 0(\bmod 4)$ and $n \equiv 1(\bmod 2)$. As $k \geq 4$, relation (66) shows that $S_{k, n}(q)=O\left(e^{-\pi \min (k-2,4) /(4 k t)}\right)=O\left(e^{-\pi /(2 k t)}\right)$; one deduces with the help of first and third relations of (54), that $\gamma_{k, n}(q)=O\left(\Gamma_{k, n}(q) e^{-\pi /(4 k t)}\right)=\varnothing$. Moreover, if $k \equiv 2$ $(\bmod 4)$ and $n \equiv 0(\bmod 2)$, relation (65) combined with the third formula of (54) implies that $\gamma_{k, n}=O\left(\Gamma_{k, n}(q) e^{-\pi /(4 k t)}\right)=\varnothing$. Thus the first statement of the theorem is obtained. If $k=4 k^{\prime}$ and $n=2 n^{\prime}$ with $k^{\prime}, n^{\prime} \in \mathbf{Z}$, one simultaneously considers the first relation of (54) and formula (65) and one finds that

$$
\gamma_{k, n}(q)=2 \Gamma_{k, n} \cos \left(n^{\prime}-k^{\prime}\right) \pi+\varnothing,
$$

which is equivalent to (69). The case of $k=4 k^{\prime}+2$ and $n=2 n^{\prime}+1$ may be treated in a similar way, using the third relation of (54) and formula (66).

In the case $k=4 k^{\prime}+1$ and $n \in\left\{2 n^{\prime}, 2 n^{\prime}+1\right\}$ one concludes directly by combining the second relation of (54) with (67) or (68).

As for the remaining cases, if $k=4 k^{\prime}-1$ and $n=2 n^{\prime}$, relation (67) and the last formula of (54) imply (70). Last, if $k=4 k^{\prime}-1$ and $n=2 n^{\prime}-1$, relation (68) and the last formula of (54) also imply (70).

Proof of theorem 2 It follows directly from theorem 3 .

\section{References}

[1] Bruce C. Berndt. Ramanujan's notebooks. Part III. New York etc.: Springer-Verlag, 1991.

[2] Hans Rademacher. Topics in analytic number theory., volume 169. Springer, Berlin, 1973.

[3] Jacques Sauloy. Théorie analytique locale des équations aux $q$-différences de pentes arbitraires. Submitted for publication; see arXiv:2006.03237v1, 2020. 
[4] Jean-Pierre Serre. A course in arithmetic. Translation of "Cours d'arithmetique". 2nd corr. print., volume 7. Springer, New York, NY, 1978.

[5] Changgui Zhang. A discrete summation for linear $q$-difference equations with analytic coefficients: General theory and examples. (Une sommation discrète pour des équations aux $q$-différences linéaires et à coefficients analytiques: Théorie générale et exemples.). Braaksma, B. L. J. (ed.) et al., Differential equations and the Stokes phenomenon. Proceedings of the conference, Groningen, Netherlands, May 28-30, 2001. Singapore: World Scientific. 309-329 (2002)., 2002. 